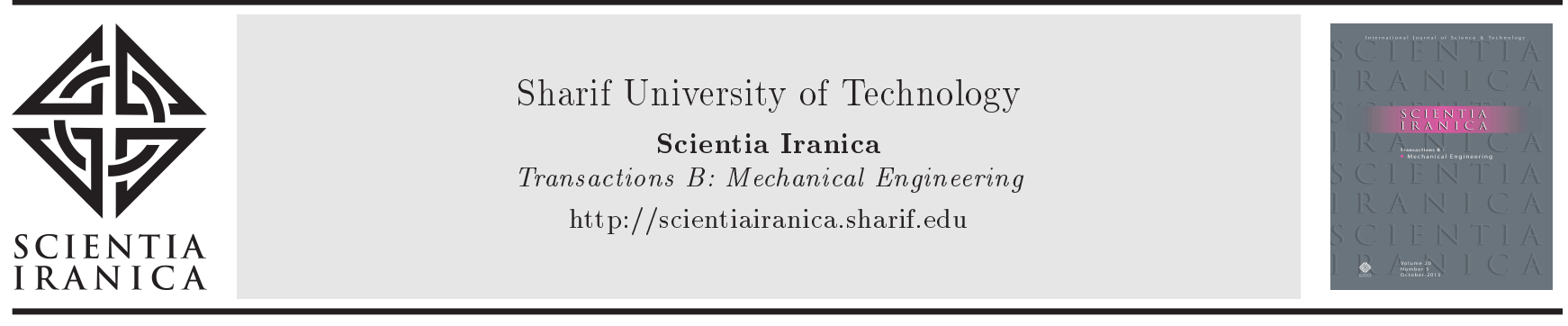

\title{
Layerwise finite element piezoelasticity analysis of functionally graded shell panel integrated with piezoelectric actuator and sensor
}

\author{
M. Javanbakht* and M. Mohammadian \\ Department of Mechanical Engineering, Isfahan University of Technology, Isfahan, P.O. Box 84156-83111, Iran.
}

Received 6 February 2017; received in revised form 20 June 2017; accepted 14 October 2017

\author{
KEYWORDS \\ Finite element; \\ Piezoelectric; \\ Functionally graded; \\ Elasticity.
}

\begin{abstract}
In the present study, a layerwise finite element method is utilized to solve the coupled elasticity and piezoelectricity equations to study a functionally graded shell panel integrated with piezoelectric layers under electromechanical loading. The system of equations is reduced to ordinary differential equations with variable coefficients by means of trigonometric function expansion in circumferential and longitudinal directions satisfying mechanical and electrical boundary conditions. These equations are solved using the Galerkin FEM and Newmark method. The results of stress, displacement, and electrical potential are presented and the effect of panel thickness and applied voltage on the structural behavior is investigated.

(C) 2018 Sharif University of Technology. All rights reserved.
\end{abstract}

\section{Introduction}

In recent years, smart materials have been broadly used in many smart structures and systems. Piezoelectric as one of the most common smart materials has been used as sensor for monitoring and as actuator for controlling the response of structures [1-7] due to its coupled mechanical and electrical properties. In comparison with other smart materials, piezoelectric reveals some important advantages such as high accuracy, generation of large forces, and very fast response. Hence, piezoelectric characteristics have been of great interest and a lot of works have been devoted to investigating its different properties in different size scales [8-13].

On the other hand, Functionally Graded Materials (FGMs) are composite materials, microscopically

*. Corresponding author. Tel.: +983133915214

E-mail address: javanbakht@cc.iut.ac.ir (M. Javanbakht)

doi: $10.24200 /$ sci.2017.4515 inhomogeneous, the mechanical properties of which continuously vary from one surface to another [14]. FGMs have many applications in aerospace, nuclear reactors, chemical plants, and turbines. More importantly, they are promising candidates for smart composites having special arrangements of layers made of various materials to display favorite characteristics [15]. Therefore, the integration of piezoelectric materials and functionally graded materials has become an important subject in the area of smart materials and structures, and many attempts have been dedicated to investigate different structures including piezoelectric materials and functionally graded materials under different conditions.

An analytical solution for functionally graded piezoelectric laminates was suggested in [16], in which the equations were developed in terms of a Stroh-like formalism assuming that the mechanical and electric properties of the material had the same exponent-law dependence on the thickness-coordinate. An analytical approach was presented in [17] for free vibrations and dynamic responses of a simply supported func- 
tionally graded doubly curved shell integrated with functionally graded piezoelectric layers, particularly using the Hamilton's principle and the third order shear deformation theory of shells. The general theoretical analysis for a hollow cylinder made of functionally graded piezoelectric material subjected to two-dimensional electromechanical load was developed in [18]. The method of solution was based on the direct method and by using the complex Fourier series, the Navier equations were solved. An analytical approach for free vibration and dynamic response of simply supported functionally graded piezoelectric cylindrical panels subjected to impulsive loads was suggested in [19]. Using the Hamilton's principle, the equations of motion based on the First-order Shear Deformation Theory (FSDT) were derived and the Maxwell's electricity equation was taken as one of the governing equations. An analytical investigation was presented [20] for the nonlinear dynamic analysis and vibrations of imperfect FG thick double curved shallow shells with piezoelectric actuators on elastic foundations subjected to the combination of electrical, thermal, mechanical, and damping loadings. The theory of plates and shells coupled with the piezoelectricity theory was applied to functionally graded piezoelectric sensor and actuator [21]. Behjat et al. [22] studied static bending, free vibration, and dynamic responses of FG piezoelectric cylindrical panels subjected to mechanical, thermal, and electrical loadings using the FSDT and the FEM. A three-dimensional elasticity solution for static analysis of a simply supported FG cylindrical panel integrated with piezoelectric layers under normal load and electric excitation was presented in [23]. The static bending, free vibration, and transient responses of functionally graded piezoelectric plates under mechanical and electrical loads were investigated in [24] based on the FSDT, Hamilton's principle, and the FEM. The FEM of functionally graded plates integrated with piezoelectric patches was presented in [25]. Dynamic analysis of functionally graded piezoelectric shell panel based on the threedimensional elasticity theory was presented in $[26,27]$. Functionally graded shallow and non-shallow shell panels with piezoelectric layers were studied under mechanical loading and electrostatic excitation [28]. Functionally graded and layered magneto-electro-elastic plates and shells were studied [29] using a semi-finite element method so that a series solution was assumed in the plane of the plate and the finite element procedure was adopted across the thickness of the plate. Also, FG shells were analyzed using a layerwise model in which cylindrical shells were assumed to include many homogeneous sub cylinders [30]. Huang used a Higher order Shear Deformation Theory (HSDT) and the general von Karman equation to formulate the governing equations of an FG plate with thermo- piezo-electric effects under thermo-electro loading [31]. The Refined Plate Theory (RPT) was applied to FG plates with piezoelectric layers for free vibration analysis [32]. Three-dimensional piezo-elasticity and the FEM were used to analyze a functionally graded plate integrated with two piezoelectric layers under electromechanical loading [33]. Nonlinear dynamic response of a piezoelastic FG plate with piezoelectric sensor and actuator was investigated [34] by establishing the nonlinear dynamic equations of the piezoelectric FG plate. The Differential Quadrature Method (DQM) was utilized for a three-dimensional thermoelastic analysis of a functionally graded cylindrical shell with piezoelectric layers [35]. Dynamic analysis of shallow doubly curved FG panels integrated with sensor/actuator piezoelectric layers was analytically investigated [36]. The total potential energy was derived based on the modified Sander's shell theory combined with the FSDT. The governing equations were established based on the Reddy's HSDT that included thermo-piezoelectric effects. By applying the Galerkin method and Runge-Kutta method, and using stress function, natural frequencies and dynamic response of FG hybrid shells were determined. A thin plate theory based finite element formulation was suggested by He et al. [37] to control the shape and vibration of an FG plate with integrated piezoelectric sensors and actuators under mechanical loading. Vibration analysis of functionally graded piezoelectric plates was studied in [38]. Thermo-electro-elastic analysis of functionally graded piezoelectric shells was presented in [39]. Jafari and coworkers [40] analyzed the nonlinear vibrations of simply supported FG circular cylindrical shells with a piezoelectric layer on the outer surface. They used the Donnell's nonlinear large deflection theory to model the nonlinear dynamics of the cylindrical shell. Post-buckling and thermal post-buckling of FG plates with surface-bonded piezoelectric actuators were investigated in [41]. Shen [42] and Shen and Noda [43] studied post-buckling of FG cylindrical shells with piezoelectric actuators in thermal environments. Shen and Liew [44] also studied post-buckling of FG cylindrical panels with piezoelectric layers under a combined action of axial compression, electrical, and thermal loadings. Non-linear thermal bending of a shear deformable FG plate with piezoelectric actuators under thermal and electrical loads was studied in $[45,46]$. Bending of piezoelectric plates with functionally graded material properties was investigated in [47]. Modeling and analysis of functionally graded materials and structures were reviewed in detail in [48$50]$.

In the present study, a coupled three-dimensional elasticity and piezoelectricity solution is presented for a finitely long, simply-supported, FG shell panel with a piezoelectric layer as actuator on the external surface 
and a piezoelectric layer as sensor in the internal surface. The novelty of the present work is that for an FG shell panel integrated with two piezoelectric layers, using a layerwise FEM, the effect of the piezoelectric layer subjected to both outer pressure and applied voltages as an actuator and the effect of the internal piezoelectric layer as a sensor on the mechanical and electrical responses are investigated for different applied voltages and different thicknesses.

Combined action of pressure and electrical loadings is applied to the external surface. The FG panel is assumed to be made of many isotropic sub panels and FG properties are obtained by a suitable arrangement of layers in a multilayer panel. Here, mechanical properties of the material have an exponent-law dependence on the thickness-coordinate and the piezoelectric layers are orthotropic. At the interface between any two neighboring layers, stress, displacement, and electric potential continuities are satisfied. The highly coupled Partial Differential Equations (PDEs) are reduced to Ordinary Differential Equations (ODEs) with variable coefficients by means of trigonometric function expansion in circumferential and longitudinal directions satisfying mechanical and electrical boundary conditions. These equations are solved using the Galerkin FEM and Newmark method for an FG shell panel with inner and outer surfaces of pure aluminum (Al) and pure alumina $\left(\mathrm{Al}_{2} \mathrm{O}_{3}\right)$, respectively. The results of stress, displacement, and electrical potential are presented and the effect of panel thickness and applied voltage on the structural behavior is investigated.

\section{System of equations}

A finitely long FG shell panel with a piezoelectric layer as actuator on the external surface (radius of $R_{0}$ ) and a piezoelectric layer as sensor in the internal surface (radius of $R_{i}$ ) is considered (Figure 1). Combined action of pressure and electrical loadings is applied to

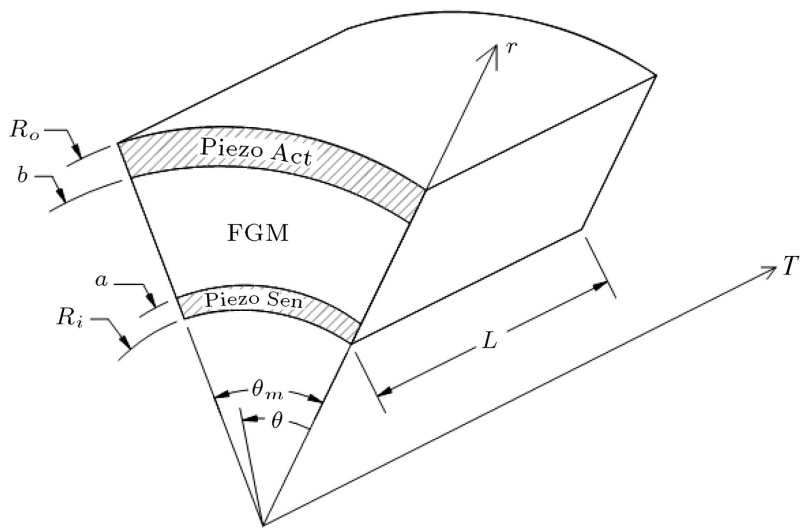

Figure 1. Geometry of the FG shell panel with piezoelectric layers. the external surface. The span of panel is $\theta_{m}$ and the length is $L$.

The cylindrical coordinate system, $r, \theta$, and $Z$, is used, where $r, \theta$, and $Z$ are the radial, circumferential, and axial directions, respectively. The corresponding displacement components are chosen as $u_{r}, u_{\theta}$, and $u_{z}$. Neglecting body forces, the equations of motion are:

$$
\sigma_{i j, j}=\rho \ddot{u}_{i}, \quad i, j=1,2,3 .
$$

The charge equation of equilibrium for electrostatics in cylindrical coordinates is:

$$
D_{i, i}=0, \quad i=1,2,3,
$$

where $D$ is the electric charge displacement. The strain-displacement relations and the electric-potential relations of the piezoelectric medium are:

$$
\begin{aligned}
\varepsilon_{i j} & =\frac{1}{2}\left(u_{i, j}+u_{j, i}\right), \\
E_{i} & =-\psi_{, i},
\end{aligned}
$$

where $E$ and $\psi$ are the electric field and the electric potential, respectively.

The linear constitutive equations (piezoelasticity) of a piezoelectric medium are given by:

$$
\sigma=\mathbf{C} \varepsilon-\mathbf{e}^{\mathrm{T}} \mathbf{E}, \quad \mathbf{D}=\mathbf{e} \varepsilon+\eta \mathbf{E},
$$

which can be extended in the following form:

$$
\begin{aligned}
& \left\{\begin{array}{l}
\sigma_{r} \\
\sigma_{\theta} \\
\sigma_{z} \\
\tau_{\theta z} \\
\tau_{r z} \\
\tau_{r \theta}
\end{array}\right\}=\left[\begin{array}{cccccc}
C_{11} & C_{12} & C_{13} & 0 & 0 & 0 \\
C_{21} & C_{22} & C_{23} & 0 & 0 & 0 \\
C_{31} & C_{32} & C_{33} & 0 & 0 & 0 \\
0 & 0 & 0 & C_{44} & 0 & 0 \\
0 & 0 & 0 & 0 & C_{55} & 0 \\
0 & 0 & 0 & 0 & 0 & C_{66}
\end{array}\right]\left\{\begin{array}{c}
\varepsilon_{r} \\
\varepsilon_{\theta} \\
\varepsilon_{z} \\
\gamma_{\theta z} \\
\gamma_{r z} \\
\gamma_{r \theta}
\end{array}\right\} \\
& -\left\{\begin{array}{ccc}
e_{33} & 0 & 0 \\
e_{31} & 0 & 0 \\
e_{32} & 0 & 0 \\
0 & 0 & 0 \\
0 & 0 & e_{24} \\
0 & e_{15} & 0
\end{array}\right\}\left\{\begin{array}{l}
E_{r} \\
E_{\theta} \\
E_{z}
\end{array}\right\} \\
& \left\{\begin{array}{l}
D_{r} \\
D_{\theta} \\
D_{z}
\end{array}\right\}=\left[\begin{array}{cccccc}
e_{33} & e_{31} & e_{32} & 0 & 0 & 0 \\
0 & 0 & 0 & 0 & 0 & e_{15} \\
0 & 0 & 0 & 0 & e_{24} & 0
\end{array}\right]\left\{\begin{array}{c}
\varepsilon_{r} \\
\varepsilon_{\theta} \\
\varepsilon_{z} \\
\gamma_{\theta z} \\
\gamma_{r z} \\
\gamma_{r \theta}
\end{array}\right\} \\
& +\left[\begin{array}{ccc}
\eta_{33} & 0 & 0 \\
0 & \eta_{11} & 0 \\
0 & 0 & \eta_{22}
\end{array}\right]\left\{\begin{array}{l}
E_{r} \\
E_{\theta} \\
E_{z}
\end{array}\right\}
\end{aligned}
$$

where the superscript $T$ stands for the transpose of a 
matrix. The components of stress, $\sigma$, strain, $\varepsilon$, electric field, $E$, and electric charge displacement, $D$, are given in the cylindrical coordinate system. $\mathbf{C}, \mathbf{e}$, and $\boldsymbol{\eta}$ are the matrices of elastic, piezoelectric, and dielectric constants of the piezoelectric materials, respectively.

Substituting Eqs. (3) and (4) into Eq. (6), stress, $\sigma$, and electric charge displacement, $D$, are obtained in terms of the mechanical displacement $u$ and the electrical potential $\psi$ :

$$
\left\{\begin{array}{l}
\sigma_{r} \\
\sigma_{\theta} \\
\sigma_{z} \\
\tau_{\theta z} \\
\tau_{r z} \\
\frac{\tau_{r \theta}}{D_{r}} \\
D_{\theta} \\
D_{z}
\end{array}\right\}=\left[\begin{array}{cccc}
c_{11} \frac{\partial}{\partial r}+\frac{c_{12}}{r} & \frac{c_{12}}{r} \frac{\partial}{\partial \theta} & c_{13} \frac{\partial}{\partial z} & e_{33} \frac{\partial}{\partial r} \\
c_{12} \frac{\partial}{\partial r}+\frac{c_{22}}{r} & \frac{c_{22}}{r} \frac{\partial}{\partial \theta} & c_{23} \frac{\partial}{\partial z} & e_{31} \frac{\partial}{\partial r} \\
c_{13} \frac{\partial}{\partial r}+\frac{c_{23}}{r} & \frac{c_{23}}{r} \frac{\partial}{\partial \theta} & c_{33} \frac{\partial}{\partial z} & e_{32} \frac{\partial}{\partial r} \\
0 & c_{44} \frac{\partial}{\partial z} & \frac{c_{44}}{r} \frac{\partial}{\partial \theta} & 0 \\
c_{55} \frac{\partial}{\partial z} & 0 & c_{55} \frac{\partial}{\partial r} & e_{24} \frac{\partial}{\partial z} \\
\frac{c_{66}}{r} \frac{\partial}{\partial \theta} & c_{66}\left(\frac{\partial}{\partial r}-\frac{1}{r}\right) & 0 & \frac{e_{15}}{r} \frac{\partial}{\partial \theta} \\
e_{33} \frac{\partial}{\partial r}+\frac{e_{31}}{r} & \frac{e_{31}}{r} \frac{\partial}{\partial \theta} & e_{32} \frac{\partial}{\partial z} & -\eta_{33} \frac{\partial}{\partial r} \\
\frac{e_{15}}{r} \frac{\partial}{\partial \theta} & e_{15}\left(\frac{\partial}{\partial r}-\frac{1}{r}\right) & 0 & -\frac{\eta_{11}}{r} \frac{\partial}{\partial \theta} \\
e_{24} \frac{\partial}{\partial z} & 0 & e_{24} \frac{\partial}{\partial r} & -\frac{\eta_{22}}{r} \frac{\partial}{\partial z}
\end{array}\right]
$$

Now, substituting Eq. (7) into Eqs. (1) and (2), the governing equations of equilibrium in terms of displacements and electrical potential can be expressed as:

$$
\begin{aligned}
& {\left[c_{11} \frac{\partial^{2}}{\partial r^{2}}+\frac{c_{11}}{r} \frac{\partial}{\partial r}-\left(\frac{c_{22}}{r^{2}}-\frac{c_{66}}{r^{2}} \frac{\partial^{2}}{\partial \theta^{2}}-c_{55} \frac{\partial^{2}}{\partial z^{2}}\right)\right] u_{r}} \\
& +\left[\frac{c_{12}+c_{66}}{r} \frac{\partial^{2}}{\partial r \partial \theta}-\frac{c_{22}+c_{66}}{r^{2}} \frac{\partial}{\partial \theta}\right] u_{\theta} \\
& +\left[\left(c_{13}+c_{55}\right) \frac{\partial^{2}}{\partial r \partial z}-\frac{c_{23}-c_{13}}{r} \frac{\partial}{\partial z}\right] u_{z} \\
& +\left[e_{33} \frac{\partial^{2}}{\partial r^{2}}+\frac{e_{33}-e_{31}}{r} \frac{\partial}{\partial r}+\frac{e_{15}}{r^{2}} \frac{\partial^{2}}{\partial \theta^{2}}+e_{24} \frac{\partial^{2}}{\partial z^{2}}\right] \psi \\
& =\rho \frac{\partial^{2} u_{r}}{\partial t^{2}}, \\
& {\left[\frac{c_{66}+c_{12}}{r} \frac{\partial^{2}}{\partial r \partial \theta}+\frac{c_{22}+c_{66}}{r^{2}} \frac{\partial}{\partial \theta}\right] u_{r}}
\end{aligned}
$$

$$
\begin{aligned}
+ & {\left[c_{66} \frac{\partial^{2}}{\partial r^{2}}+\frac{c_{66}}{r} \frac{\partial}{\partial r}\right.} \\
& \left.-\left[\frac{c_{66}}{r^{2}}-\frac{c_{22}}{r^{2}} \frac{\partial^{2}}{\partial \theta^{2}}-c_{44} \frac{\partial^{2}}{\partial z^{2}}\right)\right] u_{\theta} \\
+ & {\left[\frac{c_{23}+c_{44}}{r} \frac{\partial^{2}}{\partial z \partial \theta}\right] u_{z} } \\
+ & {\left[\frac{e_{15}+e_{31}}{r} \frac{\partial^{2}}{\partial r \partial \theta}+\frac{e_{15}}{r^{2}} \frac{\partial}{\partial \theta}\right] \psi=\rho \frac{\partial^{2} u_{\theta}}{\partial t^{2}} } \\
+c_{55}+ & \left.\left.c_{13}\right) \frac{\partial^{2}}{\partial r \partial z}+\frac{c_{23}+c_{55}}{r} \frac{\partial}{\partial z}\right] u_{r} \\
+ & {\left.\left[\frac{\left.\eta_{33} \frac{\partial^{2}}{\partial r^{2}}+\frac{\eta_{33}}{r} \frac{\partial}{\partial r}+\frac{\eta_{11}}{r^{2}} \frac{\partial^{2}}{\partial \theta^{2}}+\eta_{22} \frac{\partial^{2}}{\partial z^{2}}\right] \psi=0}{e_{33}} \frac{\partial^{2}}{\partial z \partial \theta}\right] u_{\theta}+\frac{e_{31}+e_{33}}{r} \frac{\partial}{\partial r}+\frac{e_{15}}{r^{2}} \frac{\partial^{2}}{\partial \theta^{2}}+e_{24} \frac{\partial^{2}}{\partial z^{2}}\right] u_{r} } \\
+ & {\left[\left(e_{32}+e_{24}\right) \frac{\partial^{2}}{\partial r \partial z}+\frac{e_{32}}{r} \frac{\partial}{\partial z}\right] u_{z} } \\
+ & {\left[c_{55} \frac{\partial^{2}}{\partial r^{2}}+\frac{c_{55}}{r} \frac{\partial}{\partial r}+\frac{c_{44}}{r^{2}} \frac{\partial^{2}}{\partial \theta^{2}}+c_{33} \frac{\partial^{2}}{\partial z^{2}}\right] u_{z} } \\
+ & {\left.\left[e_{24}+e_{32}\right) \frac{\partial^{2}}{\partial r \partial z}+\frac{e_{24}}{r} \frac{\partial}{\partial z}\right] \psi=\rho \frac{\partial^{2} u_{z}}{r^{2}} }
\end{aligned}
$$

The simply supported boundary conditions for mechanical and electrical problems are:

$$
\begin{aligned}
& u_{r}=\sigma_{\theta}=\tau_{z \theta}=\psi=0, \quad \text { at } \quad \theta=0, \theta_{m}, \\
& u_{r}=\sigma_{\theta}=\tau_{z \theta}=\psi=0, \quad \text { at } \quad z=0, L .
\end{aligned}
$$

On the outer surface of the shell panel are a normal traction and a voltage applied while the inner surface is traction free and voltage free. In fact, the inner surface is assumed to be resistant. Also, transverse shear stresses, $\tau_{r z}$ and $\tau_{r \theta}$, are zero on the outer and the inner surfaces. The boundary conditions can be expressed as:

$$
\begin{array}{ll}
\sigma_{r}=P_{0}(\theta, z, t), & \tau_{r \theta}=\tau_{r z}=0, \\
\psi=V_{0}, & \text { at } r=R_{o}, \\
\sigma_{r}=\tau_{r z}=\tau_{r \theta}=0, & D_{r}=0, \quad \text { at } r=R_{i} .
\end{array}
$$


Since the FG panel is assumed to be made of many isotropic sub layers, stress, displacement, and electric potential continuities at each interface between any two neighboring layers must be met. Thus, the interface conditions are expressed as:

$$
\begin{array}{ll}
\left.\left.\sigma_{r}\right)_{K}=\sigma_{r}\right)_{K+1}, & \left.\left.\tau_{r \theta}\right)_{K}=\tau_{r \theta}\right)_{K+1}, \\
\left.\left.\tau_{r z}\right)_{K}=\tau_{r z}\right)_{K+1}, & \left.\left.u_{r}\right)_{K}=u_{r}\right)_{K+1}, \\
\left.\left.u_{z}\right)_{K}=u_{z}\right)_{K+1}, & \left.\left.u_{\theta}\right)_{K}=u_{\theta}\right)_{K+1}, \\
\left.\psi)_{K}=\psi\right)_{K+1}, &
\end{array}
$$

where $K$ and $K+1$ represent the numbers of each two neighboring layers (Figure 2).

In order to solve the system of equations (Eq. (8)), these highly coupled partial differential equations are reduced to ordinary differential equations with variable coefficients by means of trigonometric function expansion in circumferential and longitudinal directions satisfying mechanical and electrical boundary conditions [27,51]. The solution satisfying the boundary conditions may be assumed as:

$$
\begin{aligned}
& u_{r}=\sum_{m=1}^{\infty} \sum_{n=1}^{\infty} \phi_{r}(r, t) \sin \left(b_{m} \theta\right) \sin \left(b_{n} z\right), \\
& u_{\theta}=\sum_{m=1}^{\infty} \sum_{n=1}^{\infty} \phi_{\theta}(r, t) \cos \left(b_{m} \theta\right) \sin \left(b_{n} z\right), \\
& u_{z}=\sum_{m=1}^{\infty} \sum_{n=1}^{\infty} \phi_{z}(r, t) \sin \left(b_{m} \theta\right) \cos \left(b_{n} z\right),
\end{aligned}
$$

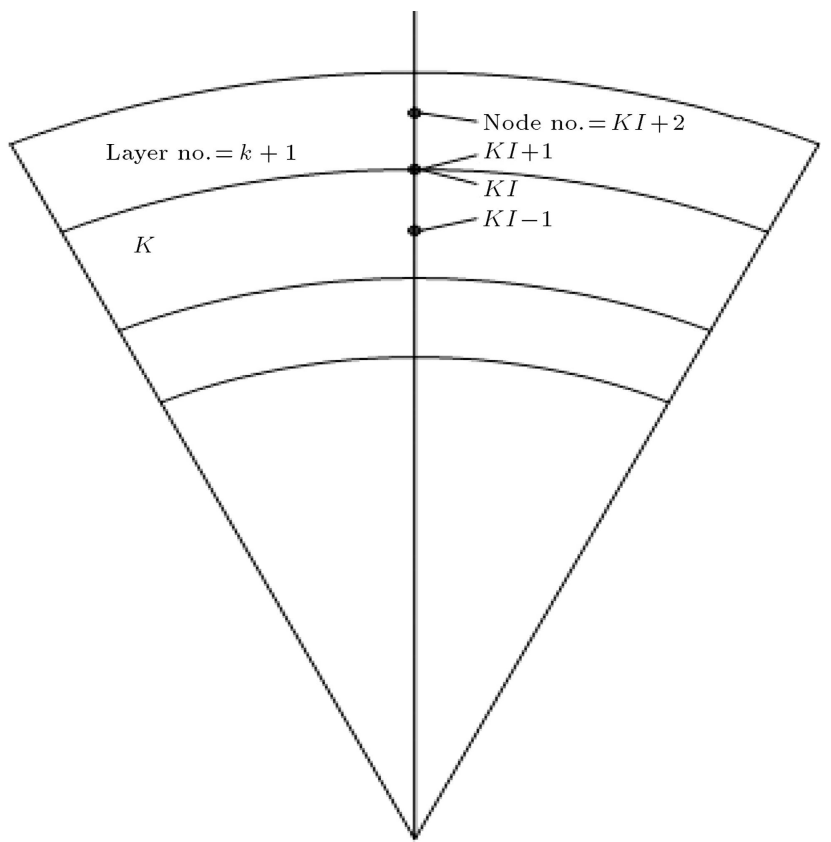

Figure 2. Configuration of the $K$ and $K+1$ layers.

$$
\psi=\sum_{m=1}^{\infty} \sum_{n=1}^{\infty} \phi_{\psi}(r, t) \sin \left(b_{m} \theta\right) \sin \left(b_{n} z\right)
$$

where:

$$
b_{m}=\frac{m \pi}{\theta_{m}}, \quad b_{n}=\frac{n \pi}{L} .
$$

Considering only the first term of the summations (i.e., $m=1, n=1$ ) in the assumed mechanical displacements and electrical potential (Eq. (12)) and substituting it into Eq. (8), the governing Partial Differential Equations (PDEs) of equilibrium in terms of displacements and electrical potential reduce to the following Ordinary Differential Equations (ODEs):

$$
\begin{aligned}
& {\left[c_{11} \frac{d^{2}}{d r^{2}}+\frac{c_{11}}{r} \frac{d}{d r}-\left(\frac{c_{22}}{r^{2}}+\frac{c_{66}}{r^{2}} b_{m}^{2}+c_{55} b_{n}^{2}\right)\right] \phi_{r}} \\
& +\left[-b_{m} \frac{c_{12}+c_{66}}{r} \frac{d}{d r}+b_{m} \frac{c_{22}+c_{66}}{r^{2}}\right] \phi_{\theta} \\
& +\left[-b_{n}\left(c_{13}+c_{55}\right) \frac{d}{d r}+b_{n} \frac{c_{23}-c_{13}}{r}\right] \phi_{z} \\
& +\left[e_{33} \frac{d^{2}}{d r^{2}}+\frac{e_{33}-e_{31}}{r} \frac{d}{d r}-\left(\frac{e_{15}}{r^{2}} b_{m}^{2}+e_{24} b_{n}^{2}\right)\right] \phi_{\psi} \\
& =\rho \frac{\partial^{2} \phi_{r}}{\partial t^{2}}, \\
& {\left[b_{m} \frac{c_{66}+c_{12}}{r} \frac{d}{d r}+\frac{c_{22}+c_{66}}{r^{2}} b_{m}\right] \phi_{r}} \\
& +\left[c_{66} \frac{d^{2}}{d r^{2}}+\frac{c_{66}}{r} \frac{d}{d r}-\left(\frac{c_{66}}{r^{2}}+\frac{c_{22}}{r^{2}} b_{m}^{2}+c_{44} b_{n}^{2}\right)\right] \phi_{\theta} \\
& +\left[-\frac{c_{23}+c_{44}}{r} b_{n} b_{m}\right] \phi_{z} \\
& +\left[\frac{e_{15}+e_{31}}{r} b_{m} \frac{d}{d r}+\frac{e_{15}}{r^{2}} b_{m}\right] \varphi_{\psi}=\rho \frac{\partial^{2} \phi_{\theta}}{\partial t^{2}}, \\
& {\left[\left(c_{55}+c_{13}\right) b_{n} \frac{d}{d r}+\frac{c_{23}+c_{55}}{r} b_{n}\right] \phi_{r}} \\
& +\left[-\frac{c_{44}+c_{23}}{r} b_{n} b_{m}\right] \phi_{\theta} \\
& +\left[c_{55} \frac{d^{2}}{d r^{2}}+\frac{c_{55}}{r} \frac{d}{d r}-\left(\frac{c_{44}}{r^{2}} b_{m}^{2}+c_{33} b_{n}^{2}\right)\right] \phi_{z} \\
& +\left[\left(e_{24}+e_{32}\right) b_{n} \frac{d}{d r}+\frac{e_{24}}{r} b_{n}\right] \phi_{\psi}=\rho \frac{\partial^{2} \phi_{z}}{\partial t^{2}},
\end{aligned}
$$




$$
\begin{gathered}
{\left[e_{33} \frac{d^{2}}{d r^{2}}+\frac{e_{31}+e_{33}}{r} \frac{d}{d r}-\left(\frac{e_{15}}{r^{2}} b_{m}^{2}+e_{24} b_{n}^{2}\right)\right] \phi_{r}} \\
+\left[-\frac{e_{31}+e_{15}}{r} b_{m} \frac{d}{d r}+\frac{e_{15}}{r^{2}} b_{m}\right] \phi_{\theta} \\
+\left[-\left(e_{32}+e_{24}\right) b_{n} \frac{d}{d r}-\frac{e_{32}}{r} b_{n}\right] \phi_{z} \\
-\left[\eta_{33} \frac{d^{2}}{d r^{2}}+\frac{\eta_{33}}{r} \frac{d}{d r}-\left(\frac{\eta_{11}}{r^{2}} b_{m}^{2}+\eta_{22} b_{n}^{2}\right)\right] \varphi_{\psi}=0 .
\end{gathered}
$$

This system of equations is solved by considering linear shape functions $N_{i}$ and $N_{j}$ for $\phi_{r}, \phi_{\theta}, \phi_{z}$, and $\phi_{\psi}$ as follows:

$$
\begin{aligned}
\phi_{s} & =\left[\begin{array}{ll}
N_{i} & N_{j}
\end{array}\right]\left[\begin{array}{l}
\phi_{s i} \\
\phi_{s j}
\end{array}\right], \quad s=r, \theta, z, \\
\phi_{\psi} & =\left[\begin{array}{ll}
N_{i} & N_{j}
\end{array}\right]\left[\begin{array}{l}
\phi_{\psi_{i}} \\
\phi_{\psi_{j}}
\end{array}\right],
\end{aligned}
$$

where:

$$
N_{i}=\frac{r_{j}-r}{r_{j}-r_{i}}, \quad N_{j}=\frac{r-r_{i}}{r_{j}-r_{i}} .
$$

Applying the formal Galerkin finite element method to the first equation of Eq. (13) yields:

$$
\begin{aligned}
& \int_{r_{i}}\left\{\begin{array}{l}
{\left[c_{11} \frac{d^{2}}{d r^{2}}+\frac{c_{11}}{r} \frac{d}{d r}-\left(\frac{c_{22}}{r^{2}}+\frac{c_{66}}{r^{2}} b_{m}^{2}+c_{55} b_{n}^{2}\right)\right] \phi_{r}} \\
+\left[-b_{m} \frac{c_{12}+c_{66}}{r} \frac{d}{d r}+b_{m} \frac{c_{22}+c_{66}}{r^{2}}\right] \phi_{\theta} \\
+\left[-b_{n}\left(c_{13}+c_{55}\right) \frac{d}{d r}+b_{n} \frac{c_{23}-c_{13}}{r}\right] \phi_{z} \\
+\left[e_{33} \frac{d^{2}}{d r^{2}}+\frac{e_{33}-e_{31}}{r} \frac{d}{d r}-\left(\frac{e_{15}}{r^{2}} b_{m}^{2}+e_{24} b_{n}^{2}\right)\right] \phi_{\psi}
\end{array}\right\} \\
& \times N_{i} d r=0 .
\end{aligned}
$$

By integrating the other three ordinary differential equations, three similar equations are obtained. Changing $N_{i}$ to $N_{j}$ and then repeating the above procedure, four other equations are obtained. The result is written in the following finite element equilibrium equation for each non-boundary element:

$$
[M]_{e}\{\ddot{X}\}_{e}+[K]_{e}\{X\}_{e}=\{F\}_{e}
$$

where $[M]_{8 \times 8}, \quad[K]_{8 \times 8}$, and $\{f\}_{8 \times 1}$ are the mass, stiffness, and force matrices, respectively, and:

$$
\{X\}_{e}^{T}=\left\{\begin{array}{llllllll}
\phi_{r i} & \phi_{\theta i} & \phi_{z i} & \phi_{\psi i} & \phi_{r j} & \phi_{\theta j} & \phi_{z j} & \phi_{\psi j}
\end{array}\right\}
$$

Applying the boundary conditions in Eqs. (9) and (10) for the first and last nodes in the inner and outer surfaces and using Eq. (16), the finite element equilibrium equations for the first and last elements become:

$$
\begin{aligned}
& {[M]_{1}\{\ddot{X}\}_{1}+[K]_{1}\{X\}_{1}=\{F\}_{1}} \\
& {[M]_{M I}\{\ddot{X}\}_{M I}+[K]_{M I}\{X\}_{M I}=\{F\}_{M I} .}
\end{aligned}
$$

Deriving the equilibrium conditions (11) in terms of displacement and electrical potential by using Eqs. (1)(5), the displacement components on the inner boundaries are obtained in terms of values at neighboring nodes. Substituting results into Eq. (16), the finite element equilibrium equations for two neighboring elements at interior $(K)$ th and $(K+1)$ th interfaces are obtained as:

$$
\begin{aligned}
& {[M]_{K}\{\ddot{X}\}_{K}+[K]_{K}\{X\}_{K}=\{0\},} \\
& {[M]_{K+1}\{\ddot{X}\}_{K+1}+[K]_{K+1}\{X\}_{K+1}=\{0\} .}
\end{aligned}
$$

Assembling Eqs. (16), (18), and (19), the general finite element equilibrium equation is obtained as:

$$
[M]\{\ddot{X}\}+[K]\{X\}=\{F\} .
$$

After establishing the finite element dynamic equation, the Newmark method is used to progress in time domain as follows:

$$
\begin{aligned}
\{X\}_{n+1}= & \{X\}_{n}+\Delta t\{\dot{X}\}_{n} \\
& +\Delta t^{2}\left\{(1 / 2-\beta)\{\ddot{X}\}_{n}+\beta\{\ddot{X}\}_{n+1}\right\} \\
\{\dot{X}\}_{n+1}= & \{\dot{X}\}_{n} \\
& +\Delta t\left\{(1-\alpha)\{\ddot{X}\}_{n}+\alpha\{\ddot{X}\}_{n+1}\right\}_{(21}
\end{aligned}
$$

where $\alpha$ and $\beta$ are chosen to be 0.25 and 0.5 , respectively, to ensure the numerical stability [27,51].

\section{Numerical results and discussion}

The piezoelectric laminate is an orthotropic material the properties of which are given as [52]: 
$C=\left[\begin{array}{cccccc}11.5 & 7.43 & 7.78 & 0 & 0 & 0 \\ 7.43 & 13.9 & 7.43 & 0 & 0 & 0 \\ 7.78 & 7.43 & 13.9 & 0 & 0 & 0 \\ 0 & 0 & 0 & 3.06 & 0 & 0 \\ 0 & 0 & 0 & 0 & 2.56 & 0 \\ 0 & 0 & 0 & 0 & 0 & 2.56\end{array}\right] \times 10^{10} \quad \mathrm{~Pa}$,

$e=\left[\begin{array}{cccccc}15.1 & -5.20 & -5.20 & 0 & 0 & 0 \\ 0 & 0 & 0 & 0 & 0 & 12.7 \\ 0 & 0 & 0 & 0 & 12.7 & 0\end{array}\right] \quad \mathrm{Cm}^{-2}$

$\eta=\left[\begin{array}{ccc}5.62 & 0 & 0 \\ 0 & 6.46 & 0 \\ 0 & 0 & 6.46\end{array}\right] \times 10^{-9} \quad$ F.m ${ }^{-1}$.

The FG shell panel material is graded through the $r$-direction. It is made of a combined ceramic-metal material the mixing ratio of which continuously and smoothly varies in the $r$-direction. The inner surface of the shell panel (radius of $a$ ) is pure metal and the outer surface (radius of $b$ ) is pure ceramic. The material distribution is shown by:

$$
P=\left(P_{c}-P_{m}\right)\left(\frac{r-a}{b-a}\right)^{n}+P_{m},
$$

where $P$ stands for material property, $n$ is a nonnegative volume fraction exponent, and subscripts $c$ and $m$ stand for ceramic and metal, respectively. In the present study, the inner surface of the shell is pure aluminum $(\mathrm{Al})$ and the outer surface is pure alumina $\left(\mathrm{Al}_{2} \mathrm{O}_{3}\right)$ the properties of which are given in Table 1.

The FG shell panel is divided to $M$ sub-shell panels ( $M$ layers) and each sub-shell panel is assumed to be isotropic so that FG properties are obtained by a suitable arrangement of layers in a multilayer panel. For example, the variation of density along the radial direction for three different exponents $n$, using Eq. (23), is shown in Figure 3.

It is found that the proper number of sub-layers is $M=50$ on which a good agreement between the present results and those from previous works is found. For $M>50$, the results have a negligible change in comparison with the results for $M=50$.

The applied pressure is chosen as:

$$
\begin{aligned}
& P_{0}(\theta, z, t)=\sum_{m=1}^{\infty} P_{0}\left(1-e^{-\alpha t}\right) \sin \left(b_{m} \theta\right) \sin \left(b_{n} z\right), \\
& \alpha=13100 .
\end{aligned}
$$

In fact, the applied pressure is a quasi-static loading of

Table 1. Material properties of $\mathrm{Al}$ and $\mathrm{Al}_{2} \mathrm{O}_{3}$.

\begin{tabular}{lcc}
\hline & $\mathrm{Al}$ & $\mathbf{A l}_{\mathbf{2}} \mathbf{O}_{\mathbf{3}}$ \\
\hline Young modulus $(\mathrm{GPa})$ & 70 & 380 \\
Mass density $\left(\mathrm{kg} / \mathbf{m}^{\mathbf{3}}\right)$ & 2702 & 3800 \\
\hline
\end{tabular}

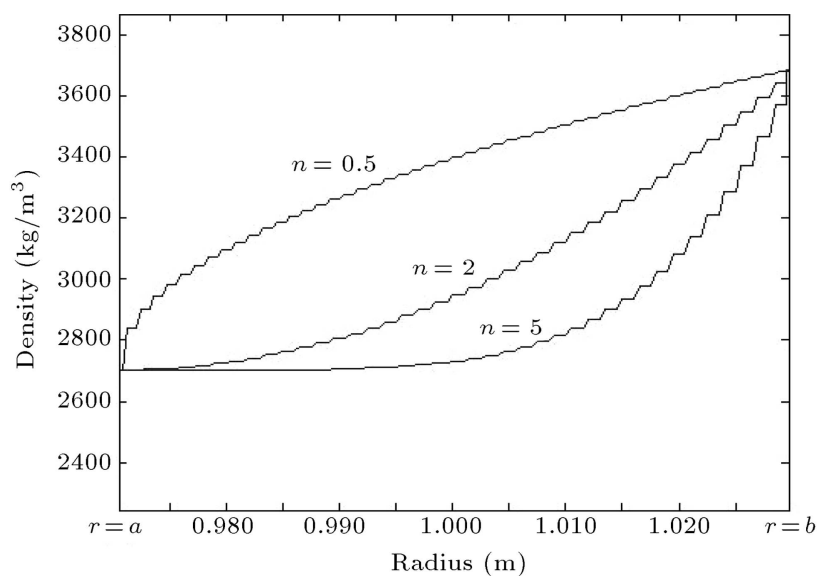

Figure 3. Variation in density of layers in the radial direction.

which the curve flattens after $t=0.001 \mathrm{~s}$ and the load reaches its peak value, $P_{0}=1 \mathrm{~Pa}$. In this respect, all the results are presented for $t=0.01 \mathrm{~s}$ as an example.

To validate the formula and numerical analysis, two different verifications are performed. First, the results of a static analysis for a [0/90] laminar composite with $S=20$ and $\theta_{m}=60 \mathrm{deg}$ are obtained and compared with those of Alibeigloo et al. [23]. As can be seen in Figures 4 and 5, there is a good agreement between our results and those in [23] and the differences are negligible.

Second, the results for an FG panel are obtained using our formula and code, and are compared with those of an exact solution suggested by Wu et al. [23]. As presented in Figures 6-11, a good agreement exists between the results and the deviations are negligible.

To present numerical results, the displacements and stresses are non-dimensionalized as follows:

$$
\begin{aligned}
& \left(\bar{u}_{r}, \bar{u}_{\theta}, \bar{u}_{z}\right)=\frac{100 \max \left(C_{i j}\right)}{H S^{4} P_{0}}\left(u_{r}, u_{\theta}, u_{z}\right), \\
& \bar{r}=\frac{r-R_{m}}{H},
\end{aligned}
$$

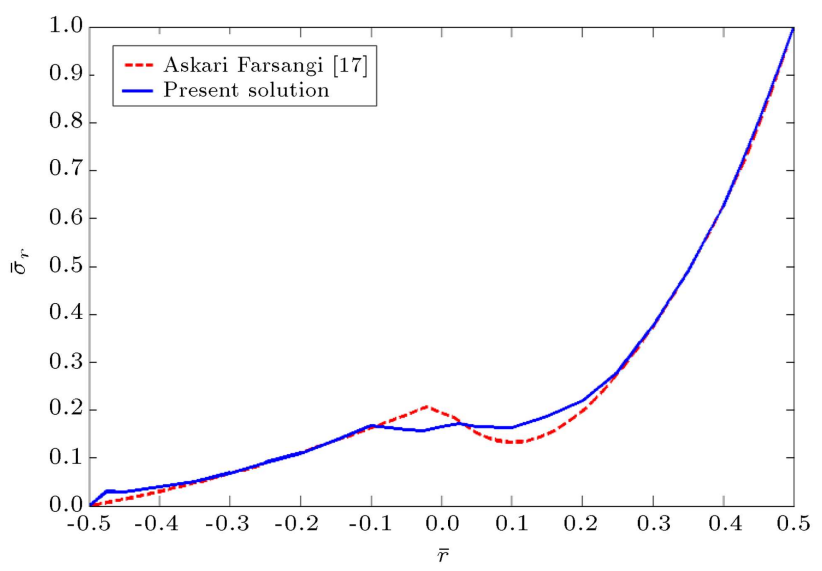

Figure 4. Distribution of $\bar{\sigma}_{r}$ across $\bar{r}$. 


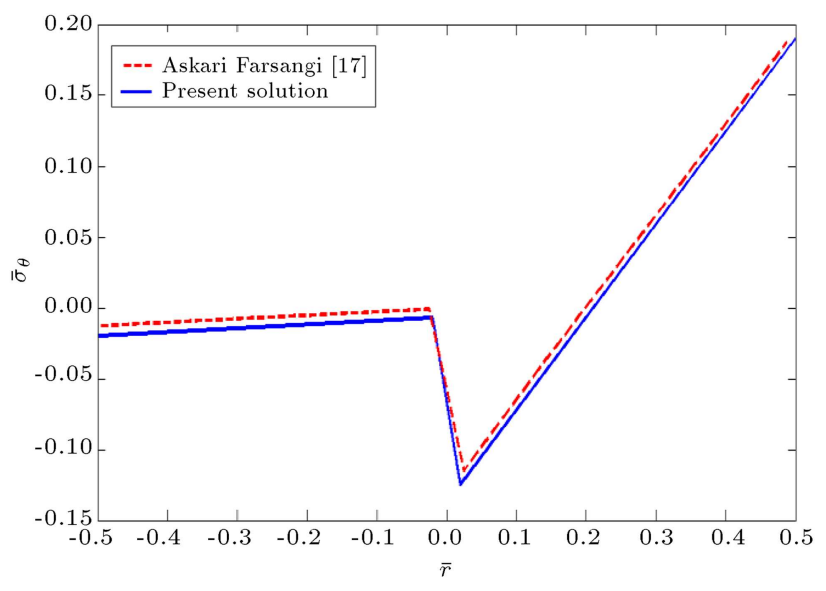

Figure 5. Distribution of $\bar{\sigma}_{\theta}$ across $\bar{r}$.

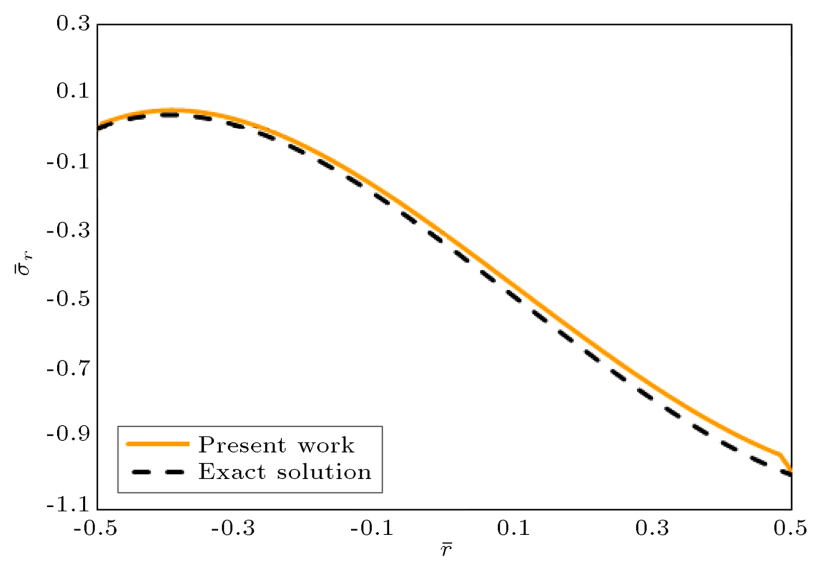

Figure 6. Distribution of $\bar{\sigma}_{r}$ across $\bar{r}(z=L / 2$, $\left.\theta=\theta_{m} / 2\right)$.

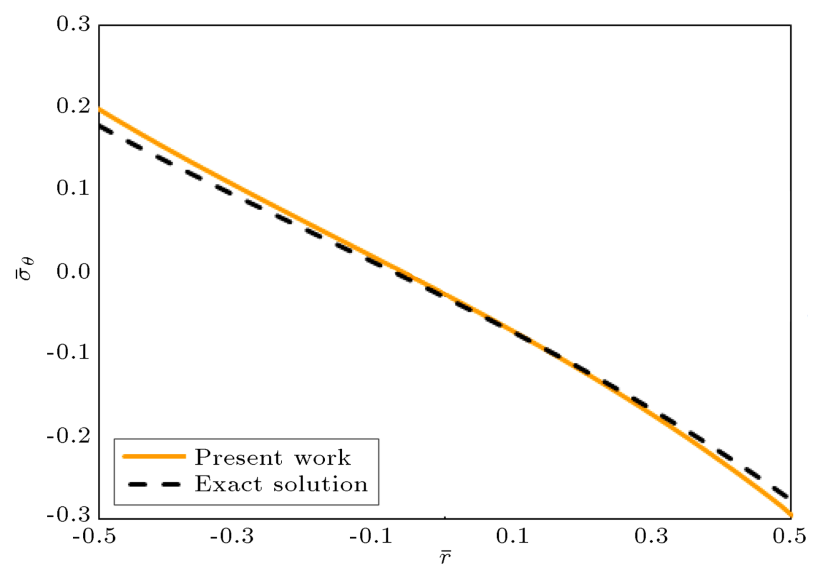

Figure 7. Distribution of $\bar{\sigma}_{\theta}$ across $\bar{r}(z=L / 2$, $\left.\theta=\theta_{m} / 2\right)$.

$\left(\bar{\sigma}_{r}, \bar{\sigma}_{\theta}, \bar{\sigma}_{z}, \bar{\tau}_{\theta z}, \bar{\tau}_{r z}, \bar{\tau}_{r \theta}\right)=\left(\sigma_{r}, \sigma_{\theta}, \sigma_{z}, \tau_{\theta z}, \tau_{r z}, \tau_{r \theta}\right) / P_{0}$,

where $S=\frac{R_{m}}{H} ; R_{m}, H$, and $C_{i j}$ are the average radius, the thickness of panel, and the component of stiffness matrix of the FG shell panel, respectively. The numerical results are presented for $R_{m}=1 \mathrm{~m}$;

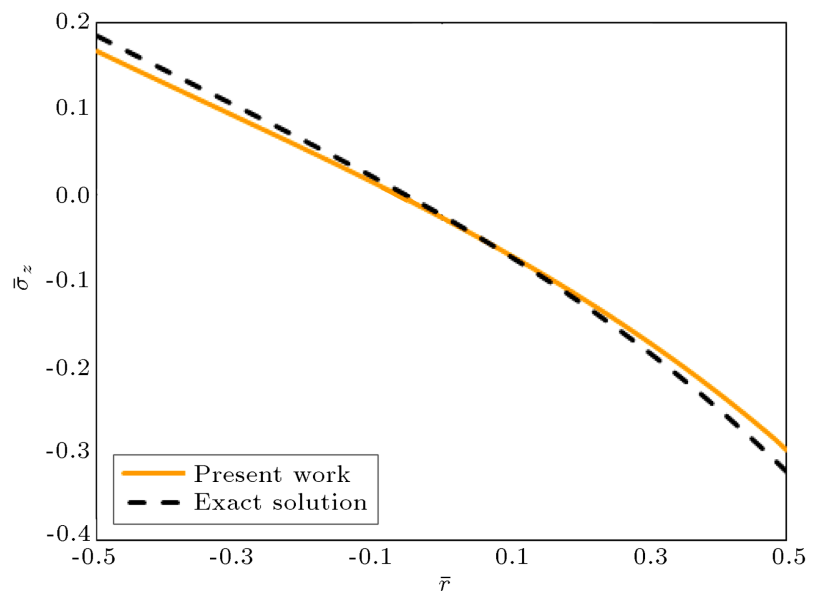

Figure 8. Distribution of $\bar{\sigma}_{z}$ across $\bar{r}\left(z=L / 2, \theta=\theta_{m}\right)$.

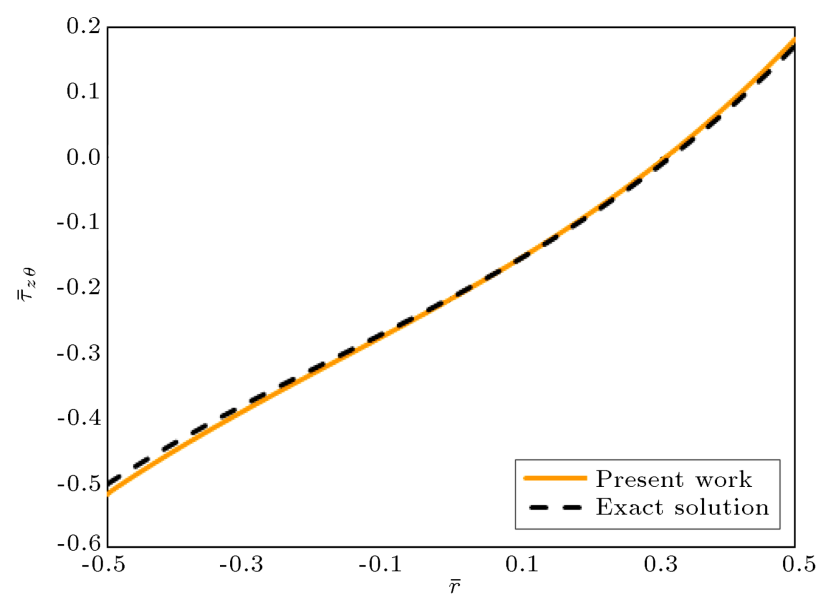

Figure 9. Distribution of $\bar{\tau}_{z \theta}$ across $\bar{r}\left(z=L / 2, \theta=\theta_{m}\right)$.

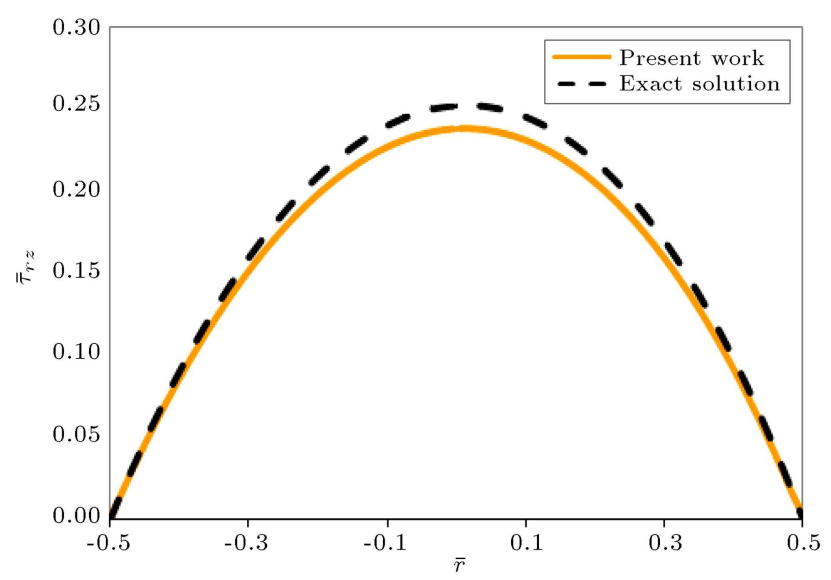

Figure 10. Distribution of $\bar{\tau}_{r z}$ across $\bar{r}(z=L / 2$, $\left.\theta=\theta_{m}\right)$.

$\theta_{m}=\pi / 3 \mathrm{rad} ; L=1 \mathrm{~m} ; P_{0}=1 \mathrm{~Pa} ; S=10,20$, and $40 ; \psi=0,0.01,0.1$, and $1 \mathrm{v}$; and $n=0.5$.

The distributions of the mechanical displacements in the radial direction are presented in Figures 12-14 for $V=0.01 \mathrm{v}$ and three different values of $S$. The radial displacement, $\bar{u}_{r}$, is almost constant across $\bar{r}$ 


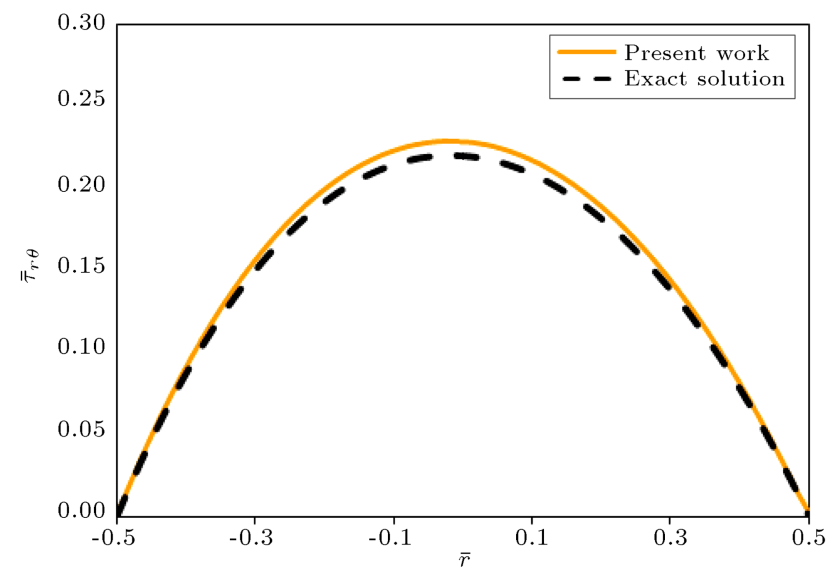

Figure 11. Distribution of $\bar{\tau}_{r \theta}$ across $\bar{r}(z=L / 2$, $\left.\theta=\theta_{m}\right)$.

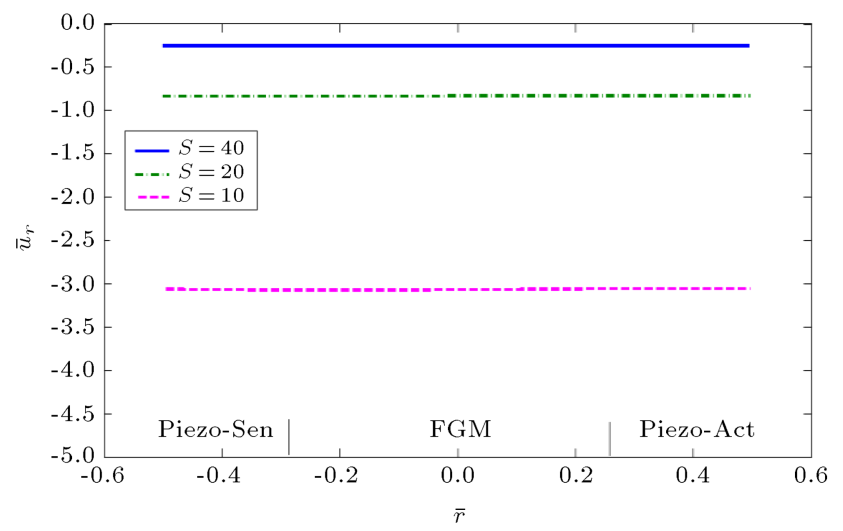

Figure 12. Distribution of $\bar{u}_{r}$ across $\bar{r}$.

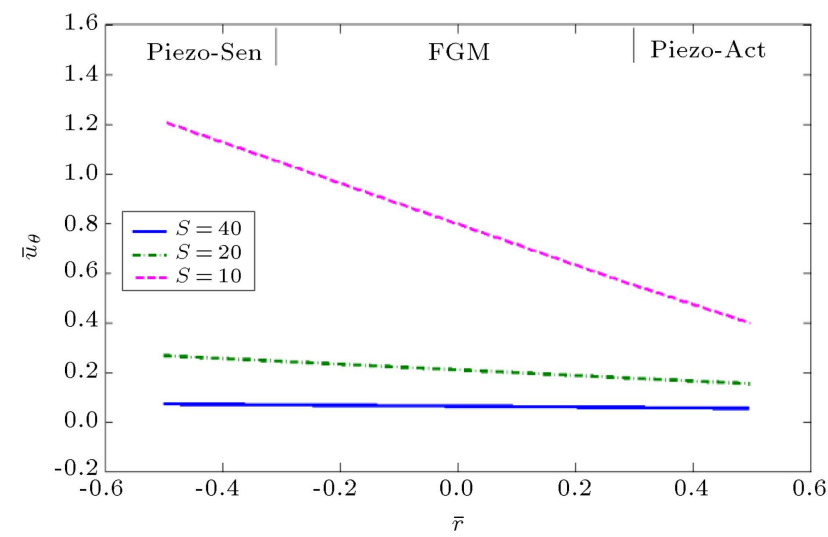

Figure 13. Distribution of $\bar{u}_{\theta}$ across $\bar{r}$.

and its magnitude increases with increasing the value of $S$. The circumferential and longitudinal displacements, $\bar{u}_{\theta}$ and $\bar{u}_{z}$, linearly vary along the radial direction, $\bar{r}$. The smaller $S$ (i.e., thicker panel), the larger the slope of the variation of $\bar{u}_{\theta}$ and $\bar{u}_{z}$. As can be seen, this slope is almost the same for the piezoelectric and FG layers. Figure 15 illustrates the distribution of electrical potential across $\bar{r}$. The voltage is applied to the outer piezoelectric layer and it acts as an actuator.

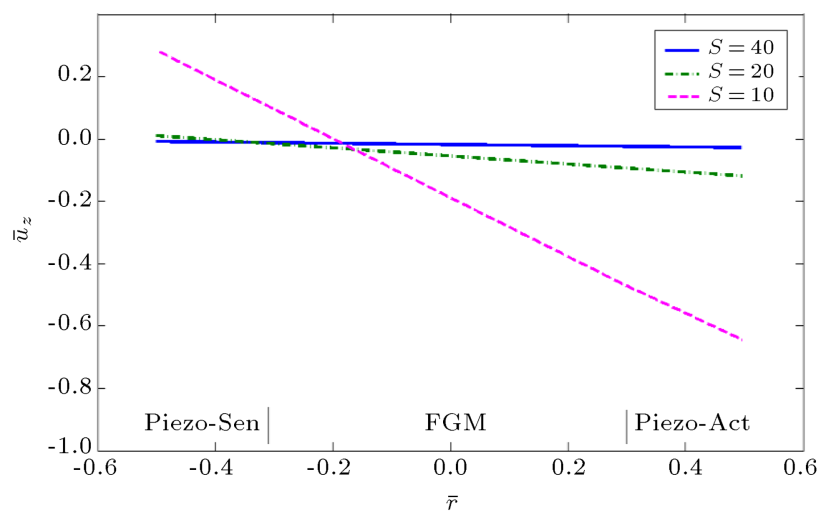

Figure 14. Distribution of $\bar{u}_{z}$ across $\bar{r}$.

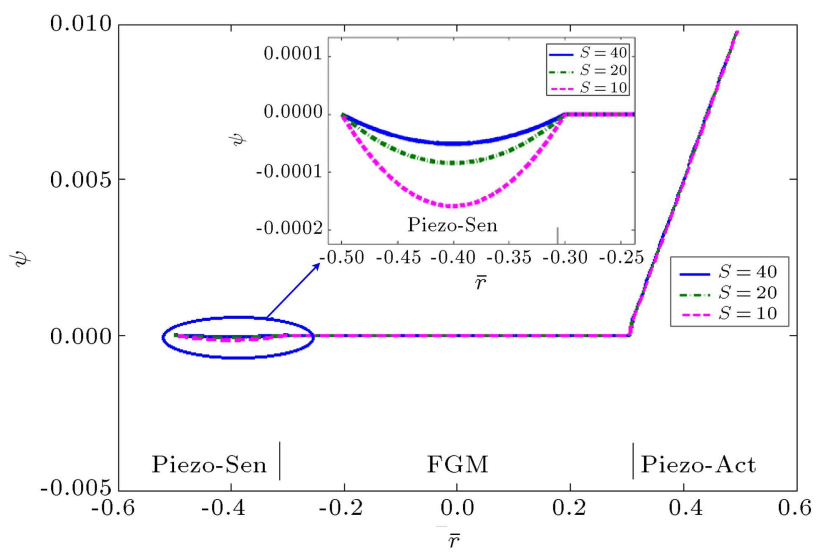

Figure 15. Distribution of $\psi$ across $\bar{r}$.

The electrical potential, $\psi$, linearly decreases from the voltage $V=0.01 \mathrm{v}$ applied to the outer surface to zero at the boundary of the piezoelectric and FG layers. The same variation of $\psi$ occurs for each value of $S$, i.e., it is independent of the piezoelectric thickness. For the inner piezoelectric layer, there is no applied voltage and it acts as a sensor. The electrical potential is obtained to be zero at the inner surface and at the boundary of the piezoelectric and FG layers. Therefore, the electrical boundary conditions and continuity are satisfied. The electrical potential varies inside the sensor in the form of a parabolic-like function across $\bar{r}$. Unlike in the actuator, the electrical potential depends on $S$ and its magnitude increases for smaller values of $S$ (thicker piezoelectric layer). Note that the magnitude of the obtained electrical potential in the sensor is much lower (two orders of magnitude) than the voltage applied to the actuator. The distributions of stress components $\bar{\sigma}_{r}, \bar{\tau}_{r \theta}$, and $\bar{\tau}_{r z}$ across $\bar{r}$ are presented in Figures 16-18. The stress $\bar{\sigma}_{r}$ at the outer surface equals the applied pressure $\left(\bar{\sigma}_{r}=-1\right)$ and is zero on the inner surface, which satisfies the mechanical boundary conditions. Also, $\bar{\tau}_{r \theta}$ and $\bar{\tau}_{r z}$ are obtained to be zero on both the inner and outer surfaces of the piezoelectric layers, in accordance with boundary conditions. All the stress components $\bar{\sigma}_{r}, \bar{\tau}_{r \theta}$, and $\bar{\tau}_{r z}$ linearly vary inside 


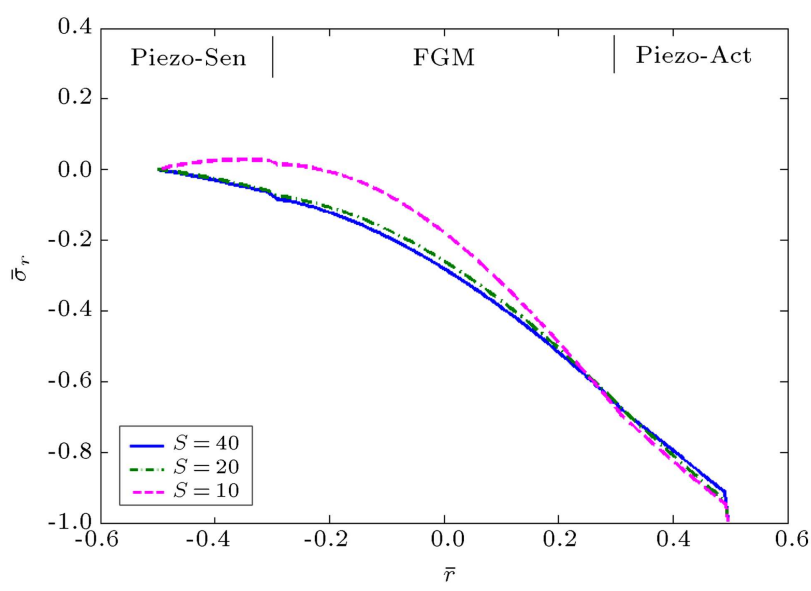

Figure 16. Distribution of $\bar{\sigma}_{r}$ across $\bar{r}$.

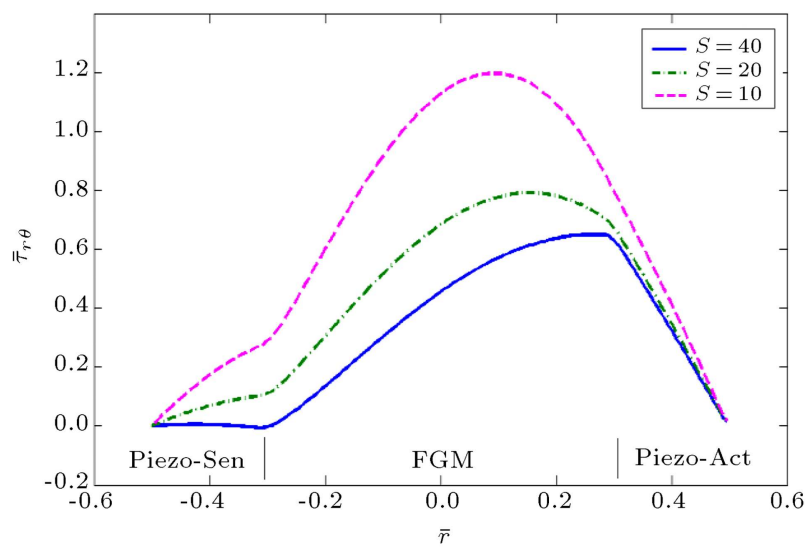

Figure 17. Distribution of $\bar{\tau}_{r \theta}$ across $\bar{r}$.

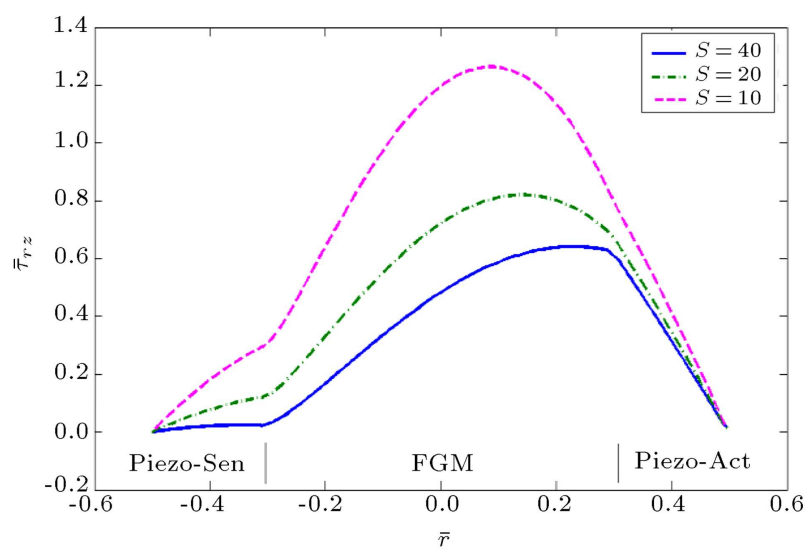

Figure 18. Distribution of $\bar{\tau}_{r} \mathrm{Z}$ across $\bar{r}$.

the actuator, while they nonlinearly vary inside the sensor and the FG panel. One more point is that these stresses are continuous across $\bar{r}$. Thus, the continuity conditions are satisfied.

The distribution of stress components $\bar{\sigma}_{\theta}, \bar{\sigma}_{z}$, and $\bar{\tau}_{\theta z}$ across $\bar{r}$ is presented in Figures 19-21. These stresses linearly vary across $\bar{r}$ inside both the actuator and sensor layers. $\bar{\sigma}_{\theta}$ and $\bar{\sigma}_{z}$ also linearly vary inside the FG panel. But, $\bar{\tau}_{\theta z}$ has a nonlinear variation in the

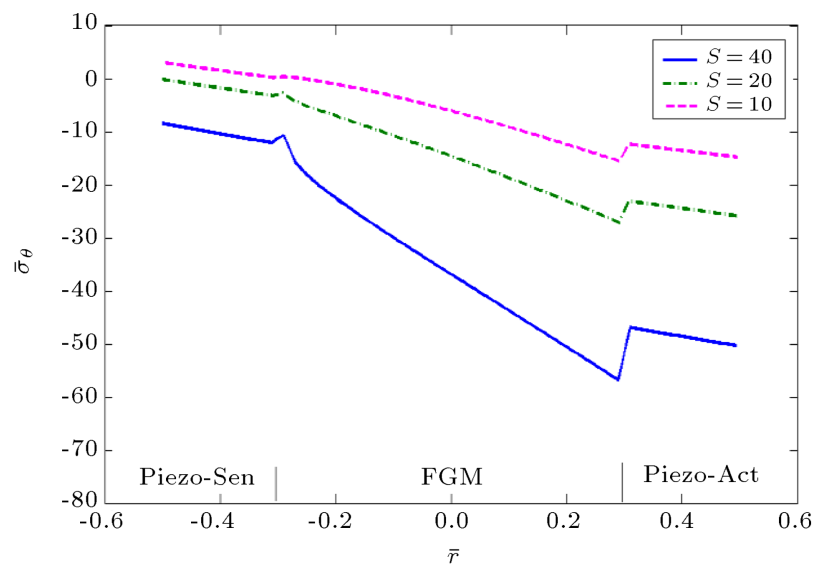

Figure 19. Distribution of $\bar{\sigma}_{\theta}$ across $\bar{r}$.

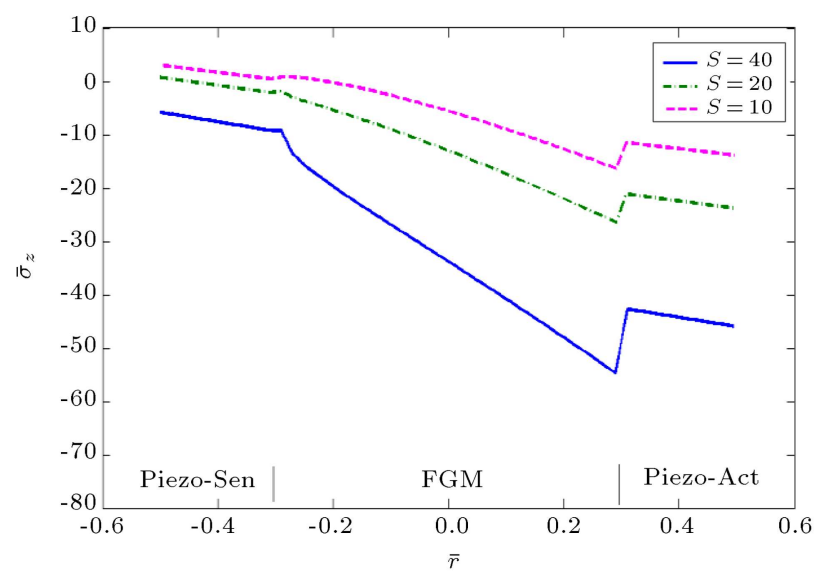

Figure 20. Distribution of $\bar{\sigma}_{z}$ across $\bar{r}$.

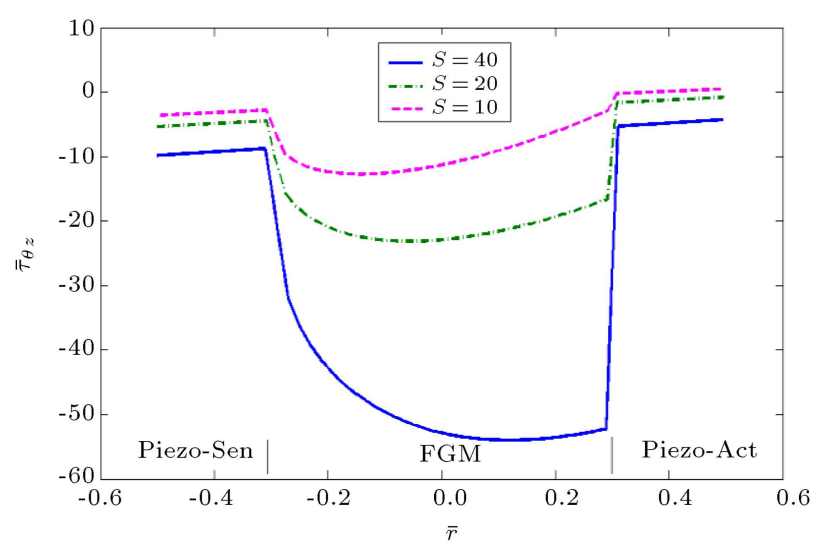

Figure 21. Distribution of $\bar{\tau}_{\theta z}$ across $\bar{r}$.

FG panel. One more point is that these stresses are not continuous across $\bar{r}$ and there are jumps in the stresses at the boundaries of the piezoelectric layers (specially the actuator) and the FG layer. This is because no continuity condition is defined for these stresses.

The effect of applied voltage to displacements and stresses is shown in Figures 22-31 for different voltages of $V=0,0.01,0.1$, and $1 \mathrm{v}$. As can be seen in Figure 22, for any applied voltage, $\bar{u}_{r}$ is almost 


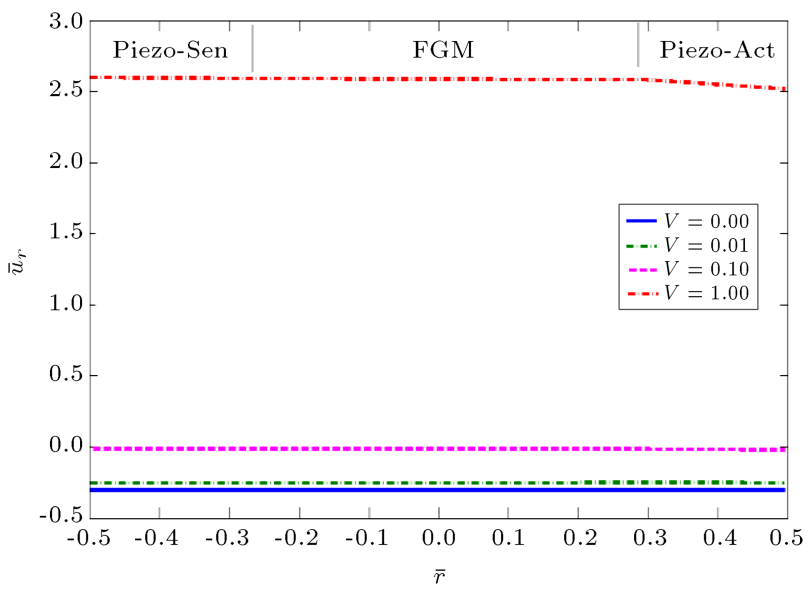

Figure 22. Distribution of $\bar{u}_{r}$ across $\bar{r}$.

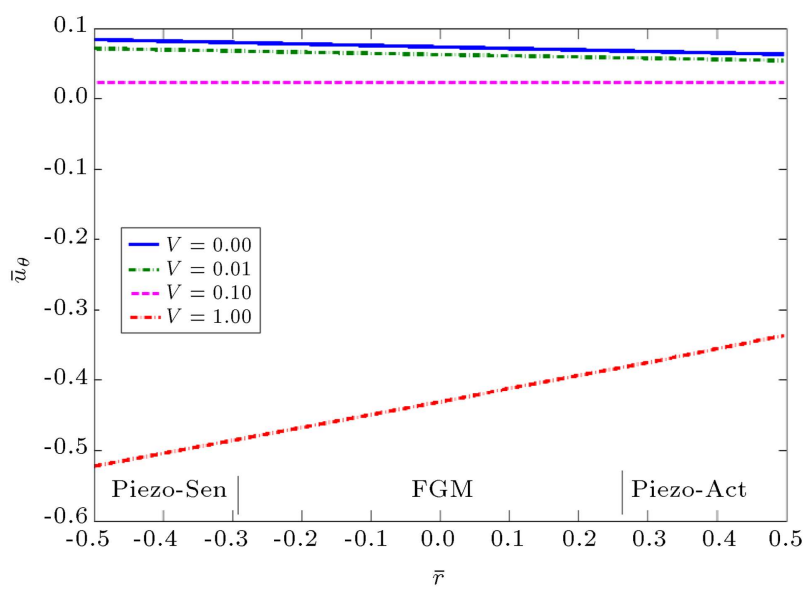

Figure 23. Distribution of $\bar{u}_{\theta}$ across $\bar{r}$.

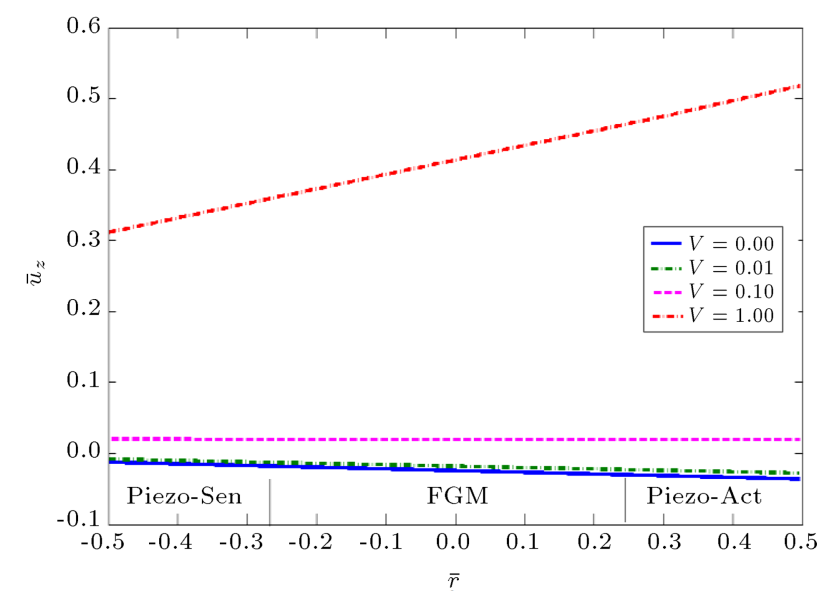

Figure 24. Distribution of $\bar{u}_{z}$ across $\bar{r}$.

constant across $\bar{r}$ in the FG and piezoelectric layers. With increasing the voltage, the compressive $\bar{u}_{r}(-0.3)$ becomes tensile and its magnitude increases (up to 2.6 for $V=1 \mathrm{v}$ ). It is found that $\bar{u}_{r}$ increases linearly in terms of the applied voltage. As shown in Figures 23 and 24 , both $\bar{u}_{\theta}$ and $\bar{u}_{z}$ linearly vary across $\bar{r}$ for any voltage and with increasing the voltage, the slopes

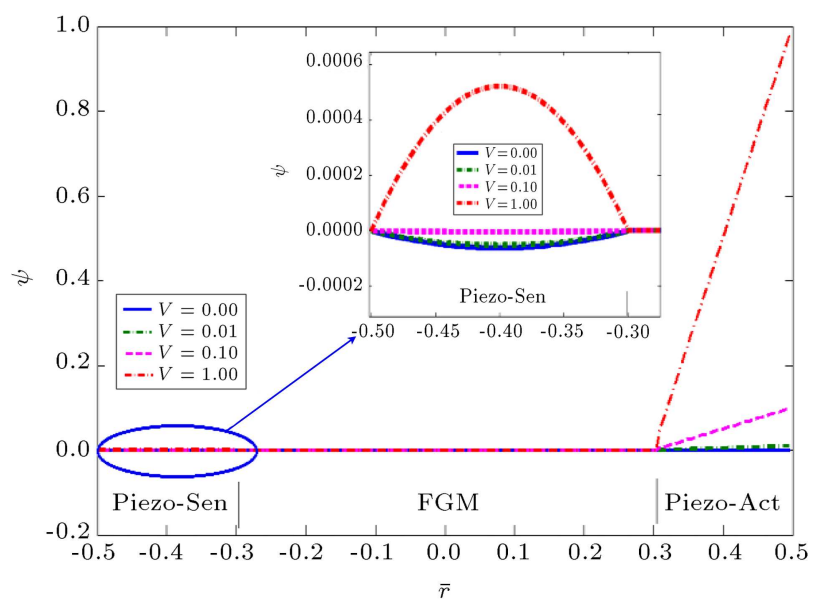

Figure 25. Distribution of $\psi$ across $\bar{r}$.

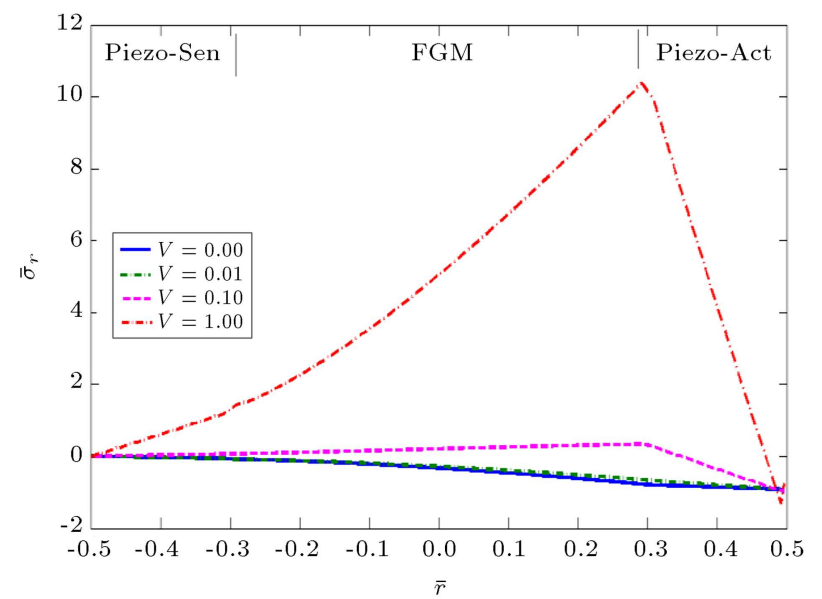

Figure 26. Distribution of $\bar{\sigma}_{r}$ across $\bar{r}$.

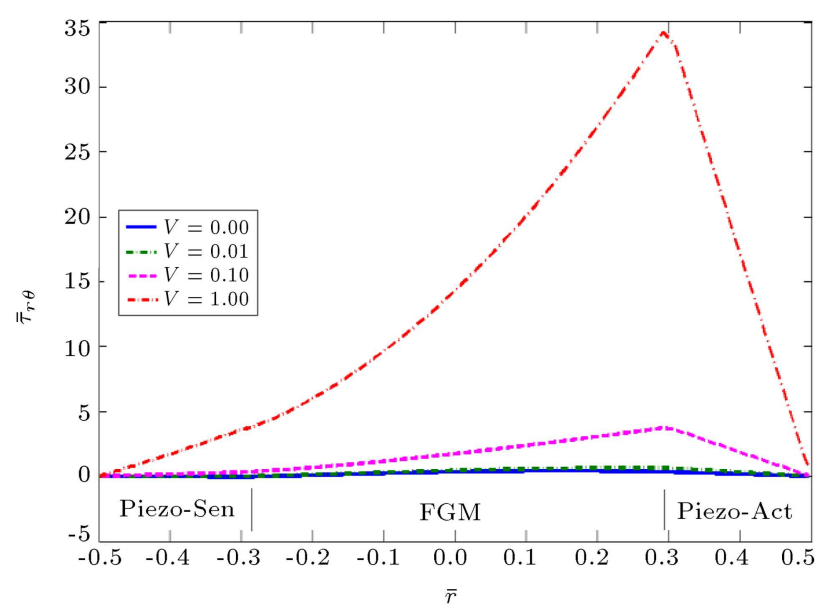

Figure 27. Distribution of $\bar{\tau}_{r \theta}$ across $\bar{r}$.

of their variations are larger and they change from compressive to tensile displacements.

The distribution of electrical potential for different applied voltages is presented in Figure 25. For any voltage, the electrical potential linearly varies from the value of voltage applied to the outer surface to zero 


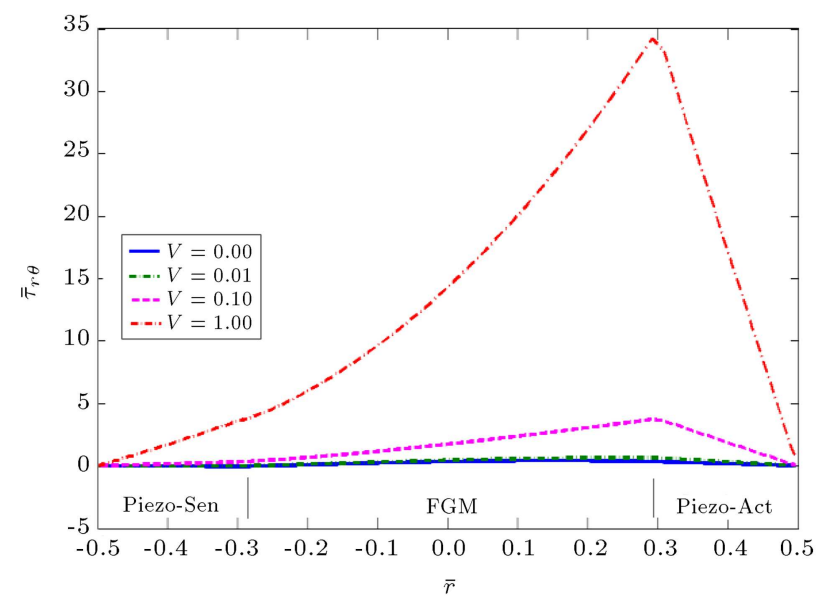

Figure 28. Distribution of $\bar{\tau}_{r z}$ across $\bar{r}$.

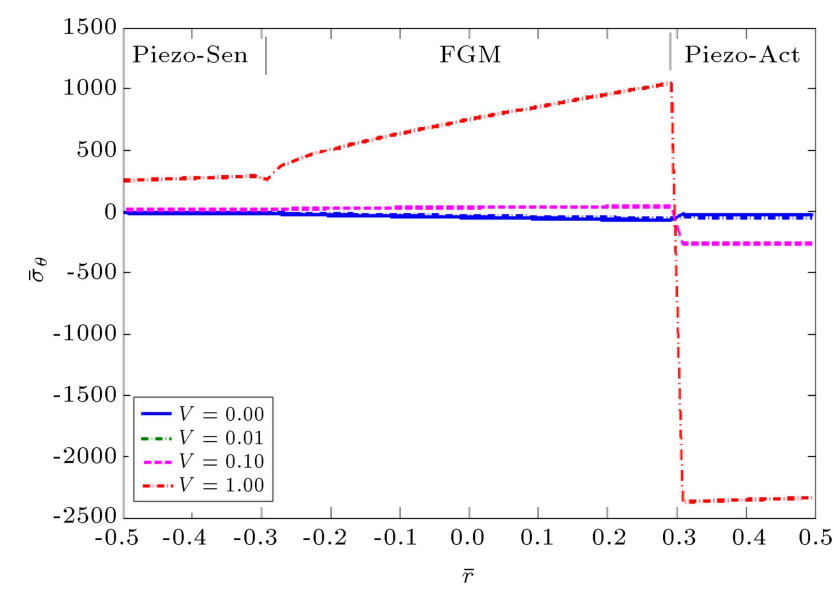

Figure 29. Distribution of $\bar{\sigma}_{\theta}$ across $\bar{r}$.

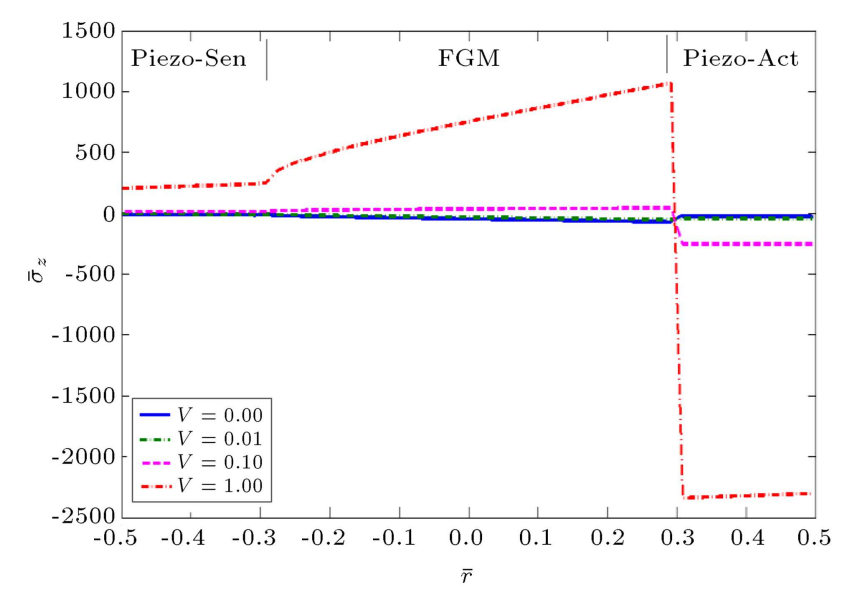

Figure 30. Distribution of $\bar{\sigma}_{z}$ across $\bar{r}$.

value at the boundary between piezoelectric (actuator) and FG layers. But, in the inner piezoelectric (sensor) layer, the electrical potential has a parabolic-like distribution with zero value at its both sides. With increasing the voltage, the sensor response changes from negative values to positive ones.

Figures 26-28 illustrate the distribution of $\bar{\sigma}_{r}$,

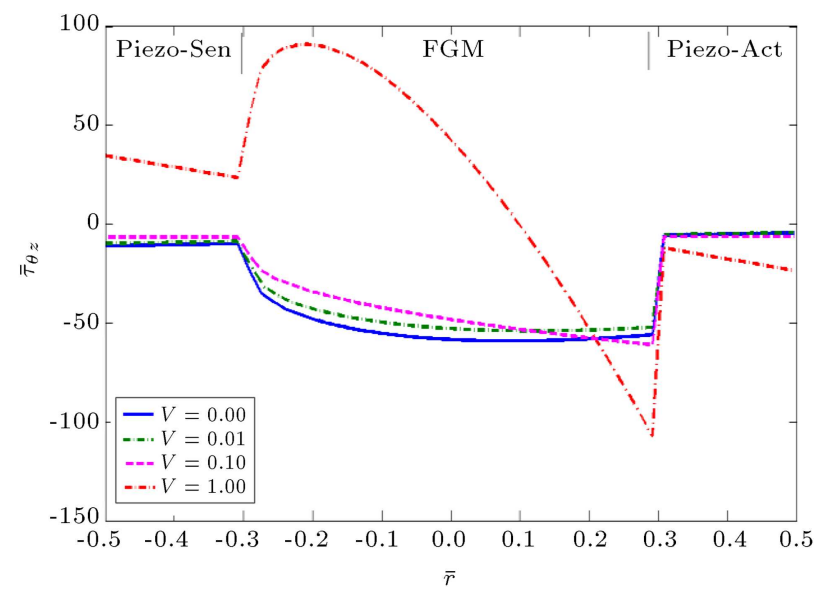

Figure 31. Distribution of $\bar{\tau}_{\theta z}$ across $\bar{r}$.

$\bar{\tau}_{r \theta}$, and $\bar{\tau}_{r z}$ across $\bar{r}$. All of these stress components linearly increase across the actuator from the outer surface to the boundary between the piezoelectric and FG layers after which they nonlinearly decrease to the inner surface. However, the variation in the inner piezoelectric (sensor) layer is quite linear. The distributions of $\bar{\sigma}_{\theta}, \bar{\sigma}_{z}$, and $\bar{\tau}_{\theta z}$ across $\bar{r}$ for different applied voltages are depicted in Figures 29-31. All of these stress components vary inside the FG and piezoelectric layers quite linearly, except for $\bar{\tau}_{\theta z}$, which varies in the FG layer nonlinearly. The larger the voltage, the larger the stresses are in the FG and piezoelectric layers. However, $\bar{\tau}_{\theta z}$ does not follow the same manner.

\section{Conclusions}

A coupled three-dimensional elasticity and piezoeletricity solution for a functionally graded shell panel with two piezoelectric layers was presented. The panel was simply supported at circumferential edges and had a finite length. The governing equations were solved using the Galerkin FEM and Newmark method. The present study showed that the Fourier series expansion in circumferential and longitudinal directions satisfying mechanical and electrical boundary conditions was suitable for the mechanical displacement and electric potential analysis. The effect of the piezoelectric layer subjected to outer pressure and applied voltages as an actuator and the effect of the internal piezoelectric layer as a sensor were investigated. The results of stress, displacement, and electrical potential were presented for different FG panel thicknesses and applied voltages, and the following conclusions could be made:

- The mechanical and electrical boundary conditions and continuity conditions were satisfied;

- For the constant applied voltage $(V=0.01 \mathrm{v})$, with increasing $S, \bar{u}_{r}$ was almost constant across $\bar{r}$ and 
its magnitude increased with increasing the value of $S$. $\bar{u}_{\theta}$ and $\bar{u}_{z}$ linearly varied across $\bar{r}$. The smaller $S$ (i.e., thicker panel), the larger the slope of the variation of $\bar{u}_{\theta}$ and $\bar{u}_{z}$;

- The electrical potential, $\psi$, linearly decreased from the voltage applied to the outer surface to zero at the boundary of the piezoelectric (actuator) and FG layers, and it was independent of the piezoelectric thickness. Inside the sensor layer, the electrical potential varied in the form of a parabolic-like function across $\bar{r}$ and it was zero at its both sides. Unlike in the actuator, the electrical potential depended on $S$ and its magnitude increased for smaller values of $S$ (thicker piezoelectric layer);

- All the stress components $\bar{\sigma}_{r}, \bar{\tau}_{r \theta}$, and $\bar{\tau}_{r z}$ linearly varied inside the actuator, while they nonlinearly varied inside the sensor and the FG panel. These stresses were continuous across $\bar{r}$;

- The stress components $\bar{\sigma}_{\theta}, \bar{\sigma}_{z}$, and $\bar{\tau}_{\theta z}$ linearly varied across $\bar{r}$ inside both the actuator and sensor layers. $\bar{\sigma}_{\theta}$ and $\bar{\sigma}_{z}$ also linearly varied inside the $\mathrm{FG}$ panel. However, $\bar{\tau}_{\theta z}$ had a nonlinear variation in the FG panel. Due to the lack of continuity conditions, these stresses were not continuous across $\bar{r}$ and there were jumps in the stresses at the boundaries of the piezoelectric layers (especially the actuator) and the FG layer;

- For any applied voltage, $\bar{u}_{r}$ was almost constant across $\bar{r}$ in the FG and piezoelectric layers. With increasing the voltage, compressive $\bar{u}_{r}$ became tensile and its magnitude increased. It was found that $\bar{u}_{r}$ increased linearly in terms of the applied voltage. Both $\bar{u}_{\theta}$ and $\bar{u}_{z}$ linearly varied across $\bar{r}$ for any voltage and with increasing the voltage, the slopes of their variations were larger and they changed from compressive to tensile displacements;

- For any voltage, electrical potential linearly varied from the value of voltage applied to the outer surface to zero value at the boundary between piezoelectric (actuator) and FG layers. However, in the inner piezoelectric (sensor) layer, the electrical potential had a parabolic-like distribution with zero value at its both sides. With increasing the voltage, the sensor response changed from negative values to positive ones;

- The stress components $\bar{\sigma}_{r}, \bar{\tau}_{r \theta}$, and $\bar{\tau}_{r z}$ linearly increased across the actuator from the outer surface to the boundary between the piezoelectric and FG layers after which they nonlinearly decreased to the inner surface. However, the variation in the inner piezoelectric (sensor) layer was quite linear;

- The stress components $\bar{\sigma}_{\theta}, \bar{\sigma}_{z}$, and $\bar{\tau}_{\theta z}$ varied inside the FG and piezoelectric layers quite linearly, except for $\bar{\tau}_{\theta z}$, which varied in the FG layer nonlinearly.
The larger the voltage, the larger the stresses were in the FG and piezoelectric layers. However, $\bar{\tau}_{\theta z}$ did not follow the same manner.

The present work provides an enhanced insight into the mechanical and electric behaviors of FG structures integrated with piezoelectric layers based on an effective layerwise FEM.

\section{Acknowledgment}

The support of Isfahan University of Technology is gratefully acknowledged.

\section{References}

1. Zhang, S.J., Li, F., and Yu, F.P. "Piezoelectric materials for cryogenic and high-temperature applications", in F.-G. Yuan, Eds., Struc. Health Monitor. (SHM) in Aerospace Struct., Woodhead Publishing, pp. 59-93 (2016).

2. Chalioris, C.E., Karayannis, C.G., Angeli, G.M., Papadopoulos, N.A., Favvata, M.J., and Providakis, C.P. "Applications of smart piezoelectric materials in a wireless admittance monitoring system (WiAMS) to Structures-Tests in RC elements", Case Studies in Const. Mater., 5, pp. 1-18 (2016).

3. Ribeiro, C., Sencadas, V., Correia, D.M., and Lanceros-Méndez, S. "Piezoelectric polymers as biomaterials for tissue engineering applications", Coll. Surf. B: Biointerfaces, 136, pp. 46-55 (2015)

4. Ho, Sh.-T. and Jan, Sh.-J. "A piezoelectric motor for precision positioning applications", Precision Eng., 43, pp. 285-293 (2016).

5. Ma, H.-K., Luo, W.-F., and Lin, J.-Y. "Development of a piezoelectric micropump with novel separable design for medical applications", Sens. and Actu. A: Physical, 236, pp. 57-66 (2015)

6. Abella, F., Ribot, J., Doria, G., Duran-Sindreu, F., and Roig, M. "Applications of piezoelectric surgery in endodontic surgery: A Literature Review", J. of Endodontics, 40, pp. 325-332 (2014).

7. Camarda, A., Romani, A., and Tartagni, M. "Piezoelectric transformers for ultra-low voltage energy harvesting applications", Procedia Eng., 87, pp. 1521-1524 (2014).

8. Zhang, J. and Meguid, S.A. "On the piezoelectric potential of gallium nitride nanotubes", Nano Energy, 12, pp. 322-330 (2015).

9. Momeni, K. "A multiscale approach to nanocomposite electrical generators", Nano Energy, 4, pp. 132-139 (2014).

10. Pan, X.H., Yu, S.W., and Feng, X.Q. "A continuum theory of surface piezoelectricity for nanodielectrics", Sci. China Phys. Mech. Astron., 54, pp. 564-573 (2011). 
11. Dai, S., Gharbi, M., Sharma, P., and Park, H.S. "Surface piezoelectricity: size effects in nanostructures and the emergence of piezoelectricity in non-piezoelectric materials", J. Appl. Phys., 110, p. 104305 (2011).

12. Momeni, K., Odegard, G.M., and Yassar, R.S. "Nanocomposite electrical generator based on piezoelectric zinc oxide nanowires", J. Appl. Phys., 110, p. 114303 (2010).

13. Momeni, K. and Mortazavi, S M.Z. "Optimal aspect ratio of zinc oxide nanowires for a nanocomposite electrical generator", J. Comput. Theor. Nanosci., 9(10), pp. 1670-1674 (2012).

14. Koizumi, M. "The concept of FGM ceramic transactions", Func. Grad. Mater., 34, pp. 3-10 (1993).

15. Moya, J.S. "Layered ceramics", Adv. Mater., 7, pp. 185-189 (1995).

16. Lu, P., Lee, H.P., and Lu, C. "Exact solutions for simply supported functionally graded piezoelectric laminates by Stroh-like formalism", J. of Compos. Struct., 72, pp. 352-363 (2006).

17. Askari Farsangi, M.A. "An analytical solution for dynamic behavior of thick doubly curved functionally graded smart panels", J. of Compos. Struct., 107, pp. 88-102 (2014).

18. Jafari Fesharaki, J., Jafari Fesharaki, V., Yazdipoor, M., and Razavian, B. "Two-dimensional solution for electro-mechanical behavior of functionally graded piezoelectric hollow cylinder", Appl. Mathematical Model., 36(11), pp. 5521-5533 (2012).

19. Bodaghi, M. and Shakeri, M. "An analytical approach for free vibration and transient response of functionally graded piezoelectric cylindrical panels subjected to impulsive loads", Compos. Struct., 94(5), pp. 17211735 (2012).

20. Duc, N.D., Quan, T.Q., and Luat, V.D. "Nonlinear dynamic analysis and vibration of shear deformable piezoelectric FGM double curved shallow shells under damping-thermo-electro-mechanical loads", Compos. Struct., 125, pp. 29-40 (2015).

21. PCY, L. and JD, Y. "Governing equations of piezoelectric plates with graded properties across the thickness", Pro Annu. IEEE. Int. Freq. Cont. Symp., pp. 623-631 (1996).

22. Behjat, B., Sadighi, M., Armin, A., Abbasi, M., and Salehi, M. "Static, dynamic and free vibration analysis of functionally graded piezoelectric panels using finite element method", J. Intell. Mater. Sys. Struct., 20(13), pp. 1635-1646 (2009).

23. Alibeigloo, A. and Chen, W.Q. "Elasticity solution for an FGM cylindrical panel integrated with piezoelectric layers", Europ. J. of Mech.-A/Solids, 29(4), pp. 714723 (2010).

24. Behjat, B., Salehi, M., Armin, A., Sadighi, M., and Abbasi, M. "Static and dynamic analysis of functionally graded piezoelectric plates under mechanical and electrical loading", Scientia Iranica, 18(4), pp. 986-994 (2011).
25. Panda, S. and Ray, M.C. "Nonlinear finite element analysis of functionally graded plates integrated with patches of piezoelectric fiber reinforced composite", Finite Elem. in Anal. and Des., 44, pp. 493-504 (2008).

26. Javanbakht, M., Daneshmehr, A.R., Shakeri, M., and Nateghi, A.R. "The dynamic analysis of the functionally graded piezoelectric (FGPM) shell panel based on three-dimensional elasticity theory", Appl. Math. Model., 36, pp. 5320-5333 (2012).

27. Javanbakht, M., Shakeri, M., and Sadeghi, S.N. "Dynamic analysis of functionally graded shell with piezoelectric layers based on elasticity", Proc. Inst. Mech. Eng. C J. Mech. Eng. Sci., 223, pp. 2039-2047 (2009).

28. Javanbakht, M., Shakeri, M., Sadeghi, S.N., and Daneshmehr, A.R. "The analysis of functionally graded shallow and non-shallow shell panels with piezoelectric layers under dynamic load and electrostatic excitation based on elasticity", Europ. J. of Mech.-A/Solids, 30, pp. 983-991 (2011).

29. Bhangale, R.K. and Ganesan, N. "Static analysis of simply supported functionally graded and layered magneto-electro-elastic plates", Int. J. of Sol. and Struct., 43, pp. 3230-3253 (2009).

30. Shakeri, M., Akhlaghi, M., and Hoseini, S.M. "Vibration and radial wave propagation velocity in functionally graded thick hollow cylinder", J. of Compos. Struc., 76, pp. 174-181 (2006).

31. Huang, X.L. and Shen, H.-S. "Vibration and dynamic response of functionally graded plates with piezoelectric actuators in thermal environments", J. of Sou. and Vib., 289, pp. 25-53 (2006).

32. Rouzegar, J. and Abad, F. "Free vibration analysis of FG plate with piezoelectric layers using four-variable refined plate theory", Thin Wall. Struct., 89, pp. 76-83 (2015).

33. Shakeri, M., Sadeghi, S.N., Javanbakht, M., and Hatamikian, H. "Dynamic analysis of functionally graded plate integrated with two piezoelectric layers, based on three dimensional elasticity solution", Proc. Inst. Mech. Eng. C J. Mech. Eng. Sci., 223, pp. 12971309 (2009).

34. Yiqi, M. and Yiming, F. "Nonlinear dynamic response and active vibration control for piezoelectric functionally graded plate", J. of Sou. and Vib., 329(11), pp. 2015-2028 (2010).

35. Akbari Alashti, R. and Khorsand, M. "Threedimensional thermo-elastic analysis of a functionally graded cylindrical shell with piezoelectric layers by differential quadrature method", Int. J. of Press. Vess. and Pipe., 88(5-7), pp. 167-180 (2011).

36. Kiani, Y., Sadighi, M., and Eslami, M.R. "Dynamic analysis and active control of smart doubly curved FGM panels", Compos. Struct., 102, pp. 205-216 (2013). 
37. He, X.Q., Ng, T.Y., Sivashankar, S., and Liew, K.M. "Active control of FGM plates with integrated piezoelectric sensors and actuators", Int. J. of Sol. and Struct., 38, pp. 1641-1655 (2001).

38. Kong, Y. and Liu, J. "Vibration confinement of thickness-shear and thickness-twist modes in a functionally graded piezoelectric plate", Acta Mech. Soli. Sin., 24(4), pp. 299-307 (2011).

39. Dehghan, M., Zamani Nejad, M., and Moosaie, A. "Thermo-electro-elastic analysis of functionally graded piezoelectric shells of revolution: Governing equations and solutions for some simple cases", Int. J. of Eng. Sci., 104, pp. 34-61 (2016).

40. Jafari, A.A., Khalili, S.M.R., and Tavakolian, M. "Nonlinear vibration of functionally graded cylindrical shells embedded with a piezoelectric layer", Thin Wall. Struct., 79, pp. 8-15 (2014).

41. Liew, K.M., Yang, J., and Kitipornchai, S. "Postbuckling of piezoelectric FGM plates subject to thermoelectro-mechanical loading", Int. J. of Sol. and Struct., 40, pp. 3869-3892 (2003).

42. Shen, H.-S. "Postbuckling of axially loaded FGM hybrid cylindrical shells in thermal environments", Compos. Sci. and Tech., 65(11-12), pp. 1675-1690 (2005).

43. Shen, H.-S. and Noda, N. "Postbuckling of pressureloaded FGM hybrid cylindrical shells in thermal environments", Compos. Struct., 77(4), pp. 546-560 (2007).

44. Shen, H.-S. and Liew, K. "Postbuckling of axially loaded functionally graded cylindrical panels with piezoelectric actuators in thermal environments", J. of Eng. Mech., 130(8), pp. 982-995 (2004).

45. Shen, H.-S. and Li, S.R. "Postbuckling of sandwich plates with FGM face sheets and temperaturedependent properties", Compos. Part B: Eng., 39, pp. 332-344 (2008).

46. Shen, H.-S. "Nonlinear thermal bending response of FGM plates due to heat conduction", Compos Part B: Eng., 38, pp. 201-215 (2007).

47. Sladek, J., Sladek, V., Stanak, P., Zhang, C., and Wünsche, M. "Analysis of the bending of circular piezoelectric plates with functionally graded material properties by a MLPG method", Eng. Struct., 47, pp. 81-89 (2013).

48. Wu, C.-P. and Liu, Y.-C. "A review of semi-analytical numerical methods for laminated composite and multilayered functionally graded elastic/piezoelectric plates and shells", Compos. Struct., 147, pp. 1-15 (2016).
49. Gupta, A. and Talha, M. "Recent development in modeling and analysis of functionally graded materials and structures progress in aerospace sciences", Prog. in Aerospace Sci., 79, pp. 1-14 (2015)

50. Thai, H.-T. and Kim, S.-E. "A review of theories for the modeling and analysis of functionally graded plates and shells", Compos. Struct., 128, pp. 70-86 (2015).

51. Daneshmehr, A. and Shakeri, M. "The response analysis of the piezoelectric shell panel actuators based on the theory of elasticity", ASME rth Bien. Conf. on Eng. Syst. Des. and Analysis, 2, Manchester, England, pp. 133-141 (2004).

52. Daneshmehr, A.R. and Shakeri, M. "Threedimensional elasticity solution of cross-ply shallow and non-shallow panels with piezoelectric sensors under dynamic load", Compos. Struct., 80, pp. 429-439 (2007).

53. Wu, X.-H., Shen, Y.-P., and Chen, C. "An exact solution for functionally graded piezothermoelastic cylindrical shell as sensors or actuators", Mater. Letters, 57, pp. 3532-3542 (2003).

\section{Biographies}

Mahdi Javanbakht received his BS and MS degrees in Mechanical Engineering from Amirkabir University of Technology (AUT) in 2004 and 2006, respectively, and his $\mathrm{PhD}$ degree in Mechanical Engineering from Iowa State University (ISU), USA, in 2013. He was a postdoctoral associate for one year in Aerospace Engineering Department at ISU. In 2014, he joined AUT as a faculty member and then moved to Isfahan University of Technology (IUT) in 2015. Currently, he is an Assistant Professor in the Mechanical Engineering Department at IUT. His interests include solid mechanics, nanomechanics and nanomaterials, and modeling of physical phenomena.

Mohammad Mohammadian received his BS degree in Mechanical Engineering with specialty in Thermofluid Engineering from University of Kashan, Kashan, Iran, in 2013. He has been recently graduated with MS degree from Isfahan University of Technology, Isfahan, Iran. His research interests include FEM, theories of plates and shells, and composite materials. 